\title{
Non Completely Solvable Systems of Complex First Order PDE's
}

\author{
C. Denson Hill (*) - Mauro Nacinovich (**)
}

ABSTRACT - We revisit the lack of local solvability for homogeneous vector fields with smooth complex valued coefficients, in the spirit of Nirenberg's three dimensional example. First we provide a short expository proof, in the case of $C R$ dimension one, with arbitrary $C R$ codimension. Next we pass to Lorenzian structures with any $C R$ codimension $\geq 1$ and $C R$ dimension $\geq 2$. Several different approaches are presented. Finally we discuss the connection with the absence of the Poincare lemma and the failure of local $C R$ embeddability, and present a global example.

Mathematics Subject Classification (2010). Primary: 35F05 Secondary: 32V05, 14M15, 17B20, 57T20

KEYwords. Complex vector fields, $C R$ manifolds

\section{Introduction}

\section{History and motivation}

Hans Lewy in [30] and Louis Niremberg in [36] gave two fundamental results in the theory of linear partial differential equations. The first showed that a non homogeneous equation for a first order partial differential operator with complex valued real analytic coefficients, but $\mathcal{C}^{\infty}$ smooth right hand side, may, in general, have no local weak solution. The

(*) Indirizzo dell'A.: Department of Mathematics, Stony Brook University Stony Brook NY 11794 (USA).

E-mail: dhilll@math.sunysb.edu

(**) Indirizzo dell'A.: Dipartimento di Matematica, II Università di Roma "Tor Vergata”, Via della Ricerca Scientifica, 00133 Roma (Italy).

E-mail: nacinovi@mat.uniroma2.it 
second, that a homogeneous equation for a first order partial differential with complex valued smooth coefficients may have no non constant weak local solutions. Both results were formulated and proved for partial differential operators in $\mathbb{R}^{3}$. A fuller understanding of [30] opened different directions of investigation (see e.g. [3, 4, 19, 37, 38]), especially from the two points of view of p.d.e. theory and of the analysis of $C R$ manifolds.

Nirenberg's example was especially relevant to the problem of embedding $C R$ manifold into complex manifolds. From this point of view, there have been two types of results. The Nirenberg example means that pseudoconvex three dimensional $C R$ hypersurfaces cannot be locally $C R$-embedded. However the existence of sufficiently many independent solutions of the tangential Cauchy-Riemann equations was shown to hold for pseudoconvex higher dimensional $C R$ hypersurfaces (see e.g. [1, 9, 12, 26, 27, 28]), and some general results were also obtained in terms of Lie algebras of vector fields (see e.g. [5, 17]). In the opposite direction, the counterexample of [36] was extended to $C R$ hypersurfaces with degenerate or non degenerate Lorentzian signature (see e.g. [14, 15, 20, 22, 23].

The results above were all obtained for the case of $C R$ hypersurfaces. For higher codimension, a crucial invariant is the scalar Levi form, which is parametrized by the characteristic codirections of the tangential CauchyRiemann complex. The first result in higher codimension on the absence of the Poincaré lemma at the place $q$ when some non degenerate scalar Levi form has $q$ positive eigenvalues was first proved in [3]. In [18] this result was extended to some cases where the scalar Levi form is allowed to degenerate. Much less is known about the $C R$-embedding of manifolds of higher $C R$ codimension. In [32] some results of [22] are extended under some supplementary conditions of the Cauchy-Riemann distribution. We also cite some partial results in $[10,11,16]$.

Here we want to reconsider some of these questions, also in the more general framework of general distributions of complex vector fields of [2].

\section{Contents of the paper}

Let $M$ be a smooth paracompact manifold of dimension $m$, and let $L_{1}, \ldots, L_{n}$ be smooth complex vector fields on $M$. In local coordinates each $L_{j}$ can be written as

$$
L_{j}=L_{j}(x, D)=\sum_{i=1}^{m} a_{j, i}(x) \frac{\partial}{\partial x_{i}},
$$


with coefficients $a_{j, i}$ which are assumed to be complex valued and $\mathcal{C}^{\infty}$ smooth. We are interested in considering local solutions of the homogeneous system

$$
L_{j} u=0, \quad \text { for } j=1, \ldots, n .
$$

When $n>1$, since every local distribution solution $u$ of (0.2) also satisfies

$$
\left[L_{j_{1}}, L_{j_{2}}\right] u=\left(L_{j_{1}} L_{j_{2}}-L_{j_{2}} L_{j_{1}}\right) u=0, \ldots,\left[L_{j_{1}},\left[L_{j_{2}},\left[\ldots, L_{j_{r}}\right]\right]\right] u=0
$$

for all sequence $j_{1}, j_{2}, \ldots, j_{r}$ with $1 \leq j_{1}, j_{2}, \ldots, j_{r} \leq n$, it is not restrictive to require that $L_{1}, \ldots, L_{n}$ satisfy the formal Cartan integrability conditions, i.e. that all commutators $\left[L_{j_{1}}, L_{j_{2}}\right]$ are linear combinations, with smooth coefficients, of $L_{1}, \ldots, L_{n}$.

When this condition is satisfied, and $L_{1}, \ldots, L_{n}$ define linearly independent tangent vectors on a neighborhood $U_{0}$ of a point $p_{0} \in M$, there are at most $m-n$ solutions $u_{1}, \ldots, u_{m-n}$ of $(0.2)$, with $d u_{1}\left(p_{0}\right), \ldots, d u_{m-n}\left(p_{0}\right)$ linearly independent. In fact this is always the case when the $L_{j}$ 's have coefficients that are real analytic in some coordinate neighborhood of $p_{0}$. Nirenberg's result in [36] shows that in general this is not true in the $\mathcal{C}^{\infty}$ case if $n=1, m=3$. A small perturbation of a vector field for which (0.2) has two analytically independent solutions changes to a vector field for which all local solutions of (0.2) are constant.

In $\S 1$ we show how this result extends to the case where $n=1$, but $m$ is allowed to be any integer larger or equal to 3. Namely, we show that the smooth complex vector fields for which (0.2) admits non locally constant solutions near some point of $M$ form a small nowhere dense set of first Baire category in the Fréchet space of complex vector fields on $M$.

This generalization of [36] was already given in [32], and our main goal is to extend in fact the results of [22] to the case of higher $C R$ codimension. We show that in general, given a smooth manifold $M$ and any locally $C R$ embeddable Lorentzian $C R$ structure on $M$, and a point $p_{0} \in M$, there is a new Lorentzian $C R$ structure, which is defined on a neighborhood of $p_{0}$ in $M$, and agrees to infinite order with the original one at $p_{0}$, which is not locally $C R$-embeddable. We also show that the corresponding system (0.2) is not completely integrable in the class $\mathcal{C}^{1}$.

In $\S 2$ we collect the notions on $C R$ manifolds that will be employed throughout the rest of the paper. In $\S 3, \S 4, \S 5, \S 6$ we prove the analog of the result of $\S 1$ for overdetermined systems by adaptations of the arguments therein. The results are weaker than those obtained for a scalar p.d.e. In fact, our constructions involve perturbations of an original system which, to keep formal integrability, employ either functions that are constant with 
respect to some variables, or, in $\S 5$, special morphisms of $C R$ manifolds, and, in the more special cases of $\S 6.7$, analytic objects, called $C R$-divisors. In general, we obtain new overdetermined systems which are only defined in small coordinate neighborhoods. In $\S 6.8$ we prove that we can globally define a new $C R$ structure on the Lorentzian real quadric $Q$ in $\mathrm{CP}^{v}$ which is not locally $C R$-embeddable at all points of a hyperplane section.

In $\S 5$ we also describe the $C R$ complexes and show in $\S 6.2$ how the technique used in the rest of the paper can be also employed to give proofs of the non validity of the Poincaré lemma different from those of $[3,4,18]$.

\section{Homogeneous equations with no nontrivial solutions}

In this section we prove a generalization to dimensions $\geq 3$ of a remarkable theorem of Nirenberg about local homogeneous solutions to a single homogeneous linear partial differential equation having smooth variable complex coefficients ([36], see also [32]). Our construction is close to the one in [22, Corollary p. 238] and the argument that completes the proof of Theorem 1.1 akin to [23] (compare p. 469 there and our (1.3)). Thus the methods in these section are not original, but we found it convenient to explain the general original construction in a simpler case, before extending and adapting it to discuss more general situations.

Here and in the following sections, $M$ will denote a smooth paracompact real manifold of dimension $m$.

We denote by $\mathfrak{X}^{\mathrm{C}}(M)$ the Fréchet space of all $\mathcal{C}^{\infty}$ complex vector fields on $M$. Note that $\mathfrak{X}^{\mathrm{C}}(M)$ includes also real vector fields on $M$. When $M$ is an open set in $\mathbb{R}^{m}$, each $L \in \mathfrak{X}^{\mathrm{C}}(M)$ can be written as

$$
L=a_{1}(x) \frac{\partial}{\partial x_{1}}+a_{2}(x) \frac{\partial}{\partial x_{2}}+\cdots+a_{m}(x) \frac{\partial}{\partial x_{m}},
$$

where $x=\left(x_{1}, x_{2}, \ldots, x_{m}\right)$, and the coefficients $a_{j}(x) \in \mathcal{C}^{\infty}(M)$ are (in general) complex valued.

THEOREM 1.1. Let $M$ be a smooth manifold of dimension $m \geq 3$. Then the set $\mathbb{5}$ of $L \in \mathfrak{X}^{\mathrm{C}}(M)$ for which there exists a non empty open subset $U$ of $M$, an $\varepsilon>0$, and a solution $u \in \mathcal{C}^{1+\varepsilon}(U)$ of $L u=0$ on $U$ with $d u(p) \neq 0$ for at least one $p \in U$, is a nowhere dense set of first (thin) Baire category.

In other words: the set of all $L$ on $M$ having the property that any $u$ with Hölder continuous first derivatives, which is a local solution to $L u=0$, 
in any neighborhood of any point, must be constant, is a dense set of the second (thick) Baire category.

First we prove a Lemma.

Lemma 1.2. Let $M$ be a smooth Riemannian manifold of dimension $m \geq 3$, and let $L_{0} \in \mathfrak{X}^{\mathrm{C}}(M)$. Then for every point $p_{0} \in M, h \in \mathbb{N}$, and $\varepsilon>0$ we can find $L \in \mathfrak{X}^{\mathrm{C}}(M)$ with

$$
\left\|L-L_{0}\right\|_{h, M}<\varepsilon \text { on } M,
$$

such that

$$
u \in \mathcal{C}^{1}(U), U^{\text {open }} \ni p_{0}, L u=0 \text { on } U \Longrightarrow d u\left(p_{0}\right)=0 .
$$

Proof. We can argue on a small coordinate patch $\Omega$ about $p_{0}$, and then, substituting $L_{0}$ by another vector field $L_{0}$ sufficiently close in the $h$-norm, we can assume that the coefficients of $L_{0}$ are real analytic in the coordinates in $\Omega$, and that

$$
L_{0}(p), \bar{L}_{0}(p),\left[L_{0}, \bar{L}_{0}\right](p) \text { are linearly independent in } \mathbb{C} T_{p} M, \forall p \in \Omega .
$$

Let $k=m-2$. By the real analyticity assumption, using the Cauchy-Kowalevski theorem, and by shrinking $\Omega$ if needed, we can find $k+1$ complex valued real analytic $z_{0}, z_{1}, \ldots, z_{m}$ on $\Omega$ with

$$
L_{0} z_{i}=0, \text { for } i=0, \ldots, k, \quad d z_{0} \wedge d \bar{z}_{0} \wedge d z_{1} \wedge \cdots \wedge d z_{k} \neq 0 \quad \text { on } \Omega .
$$

Let $x_{i}=\operatorname{Re} z_{i}, y_{i}=\operatorname{Im} z_{i}$. We can also arrange that $x_{0}, y_{0}, x_{1}, \ldots, x_{k}$ are real coordinates in $\Omega$ centered at $p_{0}$, and that $y_{i}\left(p_{0}\right)=0, d y_{i}\left(p_{0}\right)=0$ for $i=1, \ldots, k$. This preparation yields a local $C R$-embedding of $\Omega$ as a $C R$ submanifold of $C R$ dimension 1 and $C R$ codimension $k$ in $\mathbb{C}^{k+1}$, given by

$$
y_{i}=h_{i}\left(z_{0}, x\right) \text { for } i=1, \ldots, k,
$$

After multiplication by a nowhere zero function, we can take $L_{0}$ of the form

$$
L_{0}=\frac{\partial}{\partial \bar{z}_{0}}+\sum_{i=1}^{k} a_{i} \frac{\partial}{\partial x_{i}}, \quad a_{i} \in \mathcal{C}^{\infty}(\Omega) .
$$

The condition that $\left[L_{0}, \bar{L}_{0}\right]\left(p_{0}\right) \neq 0$ implies that $\partial^{2} h_{i} / \partial z_{0} \partial \bar{z}_{0} \neq 0$ at $p_{0}$ for some index $i$. Moreover, we note that we obtain new solutions of the homogeneous equation $L_{0} u=0$ by taking for $u$ any holomorphic function of $z_{0}, z_{1}, \ldots, z_{k}$. This allows us to use biholomorphic transformations to obtain that

$(*) \quad$ the real Hessian of $h_{1}\left(z_{0}, x\right)$ is positive definite in $\Omega$. 
In this way, the sets $\Omega_{r}=\left\{p \in \Omega \mid \operatorname{Im} z_{1}<r\right\}$, for $r>0$, form a fundamental system of open neighborhoods of $p_{0}$ in $M$. Set

$$
M_{\tau}=\left\{p \in \Omega \mid z_{1}=\tau\right\}, \text { for } \tau \in \mathbb{C} .
$$

Then, by $(*), M_{0}=\left\{p_{0}\right\}$, and there is an open connected neighborhood $\omega$ of 0 in $\mathbb{C}$ and a smooth real curve $\operatorname{Im} \tau=\phi(\operatorname{Re} \tau)$ in $\omega$, passing through 0 , with the properties

$$
\begin{aligned}
& M_{\tau} \subset \Omega \text { if } \tau \in \omega, \\
& M_{\tau}=\emptyset \text { if } \operatorname{Im} \tau<\phi(\operatorname{Re} \tau), \\
& M_{\tau}=\{\text { a point }\} \text { if } \operatorname{Im} \tau=\phi(\operatorname{Re} \tau), \\
& M_{\tau} \simeq S^{k} \text { if } \operatorname{Im} \tau>\phi(\operatorname{Re} \tau) .
\end{aligned}
$$

Let $\left\{D_{v}\right\}$ be a sequence of pairwise disjoint closed discs in

$$
\omega^{+}=\{\tau \in \omega \mid \operatorname{Im} \tau>\phi(\operatorname{Re} \tau)\}
$$

with centers and radii converging to 0 for $v \rightarrow \infty$. For a suitable $r_{0}>0$, all sets

$$
\omega_{r}^{+}=\{\tau \in \omega \mid \phi(\operatorname{Re} \tau)<\operatorname{Im} \tau<r\}
$$

are connected, for $0<r<r_{0}$. Set

$$
\omega_{r}^{\prime}=\omega_{r}^{+} \backslash \bigcup_{v} D_{v}, \quad \Omega_{r}^{\prime}=\left\{p \in \Omega \mid z_{1}(p) \in \omega_{r}^{\prime}\right\} .
$$

Let $u$ be a $\mathcal{C}^{1}$ solution of $L_{0}(u)=0$ on $\Omega_{r}^{\prime}$, for some $0<r<r_{0}$. For each $\tau \in \omega_{r}^{\prime}$ we define

$$
F(\tau)=\int_{M_{\tau}} u d z_{0} \wedge d z_{2} \wedge \cdots \wedge d z_{k}
$$

We claim that $F$ is holomorphic in $\omega_{r}^{\prime}$. Let indeed $\kappa$ be an arbitrary smooth simple closed curve in $\omega_{r}$. Then $\bigcup_{\tau \in \kappa} M_{\tau}$ is the boundary of a domain $N_{\kappa}$ in $\Omega$, that is diffeomorphic to the Cartesian product of a 2 -disc and a $(k-1)$-ball, and

$$
\begin{aligned}
\oint_{\kappa} F(\tau) d \tau & =\oint_{\kappa} d \tau \int_{M_{\tau}} u d z_{0} \wedge d z_{2} \wedge \cdots \wedge d z_{k}= \pm \int_{\partial N_{\kappa}} u d z_{0} \wedge d z_{1} \wedge d z_{2} \wedge \cdots \wedge d z_{k} \\
& = \pm \int_{N_{\tau}} d u \wedge d z_{0} \wedge d z_{1} \wedge \cdots \wedge d z_{k}=0
\end{aligned}
$$


because (see [29])

$$
d u \wedge d z_{0} \wedge d z_{1} \wedge \cdots \wedge d z_{k}=\left(L_{0} u\right) d \bar{z}_{0} \wedge d z_{0} \wedge d z_{1} \wedge \cdots \wedge d z_{k}=0 .
$$

By Morera's theorem, $F$ is holomorphic on $\omega_{r}^{\prime}$. Moreover, $F$ extends to a continuous function on the closure of $\omega_{r}^{\prime}$ in $\omega \cap\{\operatorname{Im} \tau<r\}$, that equals 0 for $\operatorname{Im} \tau=\phi(\operatorname{Re} \tau)$ because of (iii). It follows that $F(\tau)=0$ for $\tau \in \omega_{r}^{\prime}$.

For each $v \in \mathbb{N}$, let $Q_{v}=\left\{p \in \Omega \mid z_{1}(p) \in D_{v}\right\}$. We fix smooth functions $\psi_{i}$ in $\Omega$, such that $\psi_{i} d z_{0} \wedge d \bar{z}_{0} \wedge \cdots \wedge d z_{k}$ is, for each $i=0,1, \ldots, k$, a nonnegative real regular measure, with

$$
\operatorname{supp} \psi_{i}=\bigcup_{j=0}^{\infty} Q_{i+j(k+1)}
$$

and such that, for

$$
L=L_{0}+\psi_{0} \frac{\partial}{\partial z_{0}}+\sum_{i=1}^{k} \psi_{i} \frac{\partial}{\partial x_{i}}
$$

we have $\left\|L-L_{0}\right\|_{h}<\varepsilon$. Assume now that $u \in \mathcal{C}^{1}\left(\Omega_{r}\right)$ satisfies $L u=0$. Hence, for all $v$ sufficiently large, $Q_{v} \subset \Omega_{r}$, and

$$
\begin{aligned}
0 & = \pm \oint_{\partial D_{v}} d \tau \int_{M_{\tau}} u d z_{0} \wedge d z_{2} \wedge \cdots \wedge d z_{k}=\int_{\partial Q_{v}} u d z_{0} \wedge d z_{1} \wedge \cdots \wedge d z_{k} \\
& =\int_{Q_{v}}\left(L_{0} u\right) d z_{0} \wedge d \bar{z}_{0} \wedge d z_{1} \wedge \cdots \wedge d z_{k}=\int_{Q_{v}}\left(\left(L_{0}-L\right) u\right) d z_{0} \wedge d \bar{z}_{0} \wedge d z_{1} \wedge \cdots \wedge d z_{k}
\end{aligned}
$$

implies, by the mean value theorem, that, for all large $i \in \mathbb{N}$, there are points $p_{i}, p_{i}^{\prime} \in Q_{j}$ such that, for large $j$,

$$
\left\{\begin{array}{l}
\operatorname{Re} \frac{\partial u\left(p_{j(1+k)}\right)}{\partial z_{0}}=0, \operatorname{Im} \frac{\partial u\left(p_{j(1+k)}^{\prime}\right)}{\partial z_{0}}=0, \\
\operatorname{Re} \frac{\partial u\left(p_{i+j(k+1)}\right)}{\partial x_{i}}=0, \operatorname{Im} \frac{\partial u\left(p_{i+j(k+1)}^{\prime}\right)}{\partial x_{i}}=0, \text { for } i=1, \ldots, k .
\end{array}\right.
$$

By passing to the limit, as $p_{j} \rightarrow p_{0}$, we obtain that

$$
\frac{\partial u\left(p_{0}\right)}{\partial z_{0}}=0, \frac{\partial u\left(p_{0}\right)}{\partial x_{i}}=0, \text { for } i=1, \ldots, k .
$$

Together with $L u\left(p_{0}\right)=0$, this yields $d u\left(p_{0}\right)=0$.

Proof of Theorem 1.1. We fix a Riemannian metric on $M$, so that we can compute the length of vectors and covectors and the $\mathcal{C}^{h}$-norms of 
functions defined on subsets of $M$. Then we have seminorms which endow $\mathfrak{X}(M)$ with a Fréchet space topology, and we may discuss Baire category. Let $\left\{U_{v}\right\}_{v \in \mathbb{N}}$ be a countable basis of non empty open subsets of $M$, and for each $v \in \mathbb{N}$ fix a point $p_{v} \in U_{v}$. For $h \in \mathbb{N}$ we define $\mathfrak{S}(v, h)$ to be the closure in $\mathfrak{X}^{\mathrm{C}}(M)$ of the set of $L$ such that

$$
\exists u \in \mathcal{C}^{1+\frac{1}{h}}\left(U_{v}\right) \text { with }\left\{\begin{array}{l}
L u=0 \text { on } U_{v}, \\
\|u\|_{1+\frac{1}{h}, U_{v}} \leq h, \\
\left|d u\left(p_{v}\right)\right| \geq \frac{1}{h} .
\end{array}\right.
$$

The set $\mathbb{5}(v, h)$ has an empty interior. This can be proved by contradiction. If some $\mathfrak{S}(v, h)$ had an interior point, by Lemma 1.2 it would contain an interior point $L$ satisfying (1.2) with $p_{0}=p_{v}$. By definition, there is a sequence $\left\{L_{j}\right\}_{j \in \mathbb{N}}$ with $L_{j} \rightarrow L$ in $\mathfrak{X}^{\mathrm{C}}(M)$ for $j \rightarrow \infty$ such that for each $j$, there is $u_{j} \in \mathcal{C}^{1+\frac{1}{h}}\left(U_{v}\right)$ with $L_{j} u_{j}=0$ on $U_{v},\left\|u_{j}\right\|_{1+\frac{1}{h}, U_{v}} \leq h$ and $\left|d u\left(p_{v}\right)\right| \geq \frac{1}{h}$. By the Ascoli-Arzelà theorem, passing to a subsequence we can assume that $u_{j} \rightarrow u \in \mathcal{C}^{1+\frac{1}{h}}\left(U_{v}\right)$, uniformly with their first derivatives on every compact neighborhood of $p_{v}$ in $U_{v}$. Then $L u=0$ on $U_{v}$, and $\left|d u\left(p_{v}\right)\right| \geq \frac{1}{h}>0$ contradicts (1.2).

Therefore the union $\bigcup_{v, h} \mathfrak{S}(v, h)$ is a countable union of closed subsets having empty interior, hence of first Baire category. Then also 5 is of first Baire category, because $\mathfrak{S} \subset \bigcup_{v, h} \mathfrak{S}(v, h)$. This completes the proof of the
Theorem.

\section{Involutive systems and $C R$ manifolds}

To extend the result of $\S 1$ to overdetermined systems of homogeneous first order p.d.e.'s, we will develop ideas from $[14,15,20]$. In this section we begin by describing the general framework. In the following, $M$ will denote a $\mathcal{C}^{\infty}$-smooth manifold of real dimension $m$.

\section{1 - Generalized complex distributions and CR structures}

Let $\mathscr{Z}$ be a generalized distribution of smooth complex vector fields on $M$. This means that $\mathscr{Z}$ defines, for each open subset $U$ of $M$ a, $\mathcal{C}^{\infty}(U)$ submodule of $\mathfrak{X}^{\mathrm{C}}(U)$, in such a way that the assignment $U \rightarrow \mathscr{Z}(U)$ is a sheaf:

(1) If $U^{\text {open }} \subset V^{\text {open }} \subset M$, then $\left.Z\right|_{U} \in \mathscr{Z}(U)$ for all $Z \in \mathscr{Z}(V)$;

(2) If $\left\{U_{v}\right\}$ is a family of open subsets of $M$, a smooth complex vector 
field $Z$, defined on $\bigcup_{v} U_{v}$, belongs to $\mathscr{Z}\left(\bigcup_{v} U_{v}\right)$ if and only if $\left.Z\right|_{U_{v}} \in \mathscr{Z}\left(U_{v}\right)$ for all $v$.

Our main interest in the sequel will be focused on the local solutions to the homogeneous system

$$
Z u=0, \quad \forall Z \in \mathscr{Z} .
$$

It is therefore natural to assume in the following that $\mathscr{Z}$ is involutive, or formally integrable. This means that

$$
\left[Z_{1}, Z_{2}\right] \in \mathscr{Z}(U), \forall Z_{1}, Z_{2} \in \mathscr{Z}(U), \quad \forall U^{\text {open }} \subset M .
$$

Since $\mathscr{Z}$ is a fine sheaf, every germ $Z_{(p)}$ of $\mathscr{Z}$ at a point $p \in M$ is the restriction of a global $Z \in \mathscr{Z}(M)$. Thus we can for simplicity utilize global sections $Z \in \mathscr{Z}(M)$ in most of the discussion below.

For each point $p \in M$, we consider the set

$$
\boldsymbol{Z}_{p} M=\{Z(p) \mid Z \in \mathscr{Z}(M)\} \subset \mathbb{C} T_{p} M .
$$

If the dimension of the C-linear space $\boldsymbol{Z}_{p} M$ is constant, we say that $\mathscr{Z}$ is a distribution of complex vector fields.

Definition 2.1. A $C R$ structure on $M$ is the datum of an involutive distribution $\mathscr{Z}$ of smooth complex vector fields with $\mathscr{Z} \cap \overline{\mathscr{Z}}=0$.

The constant dimension $n$ of $\boldsymbol{Z}_{p} M$ is its $C R$ dimension, and $k=m-2 n$ its $C R$ codimension. We call the pair $(n, k)$ the type of the $C R$ manifold $M$.

In the case where $\mathscr{Z}$ is a $C R$ structure on $M$, we write sometimes $T^{0,1} M$ for $\boldsymbol{Z} M$.

When $M$ is a real smooth submanifold of a complex manifold $\mathbb{X}$, we consider on $M$ the generalized distribution

$$
\mathscr{Z}(U)=\left\{Z \in \mathfrak{X}^{\mathrm{C}}(U) \mid Z_{p} \in T_{p}^{0,1} \mathbb{X}, \forall p \in U\right\},
$$

where $T^{0,1} \mathbb{X}$ is the bundle of anti-holomorphic complex tangent vectors to $\mathbb{X}$. Then $\mathscr{Z}$ is involutive and $\mathscr{Z} \cap \overline{\mathscr{Z}}=0$. When $\mathscr{Z}$ has constant rank, $\mathscr{Z}$ defines a $C R$ structure on $M$, for which we say that $M$ is a $C R$-submanifold of $\mathbb{X}$.

Let $1 \leq a \leq \infty$. A complex $C R$-immersion of class $\mathcal{C}^{a}$ of $M$ is a $\mathcal{C}^{a}$ smooth immersion $\phi: M \rightarrow \mathbb{X}$ of $M$ into a complex manifold $\mathbb{X}$ with $d \phi\left(\boldsymbol{Z}_{p} M\right) \subset T_{\phi(p)}^{0,1} \mathbb{X}$ for all $p \in U$.

For any open set $U$ of $M$ we set

$$
\mathcal{O}_{M}(U)=\left\{u \in \mathcal{C}^{1}(U) \mid Z u=0, \forall Z \in \mathscr{Z}(M)\right\} .
$$

The assignment $U^{\text {open }} \rightarrow \mathcal{O}_{M}(U)$ defines a sheaf of rings of germs of complex valued differentiable functions on $M$. 


\section{2 - The differential ideal and complete integrability}

Let $\Omega_{M}^{*}=\underset{0 \leq p \leq m}{\bigoplus} \Omega_{M}^{p}$ be the sheaf of germs of alternated smooth differential forms on $M$. We associate to $\mathscr{Z}$ the differential ideal

$$
\mathcal{J}_{M}=\bigoplus_{p \geq 1} \mathcal{J}_{M}^{p} \text { with } \mathcal{J}_{M}^{p}=\left\{\eta \in \Omega_{M}^{p}|\eta|_{\mathscr{L}(M)}=0\right\} .
$$

This is a graded ideal sheaf of $\Omega_{M}^{*}$, generated by its elements of degree 1 . Being interested in the local solutions to (2.1), we can assume that $\mathcal{J}_{M}$ is complete and that $\mathscr{Z}$ is the characteristic system of $\mathcal{J}_{M}$, i.e. that

$$
\begin{aligned}
\mathscr{Z}(U) & \left.=\left\{Z \in \mathfrak{X}^{\mathrm{C}}(M) \mid Z\right\rfloor \mathcal{J}_{M}(U) \subset \mathcal{J}_{M}(U)\right\} \\
& =\left\{Z \in \mathfrak{X}^{\mathrm{C}}(U) \mid \eta(Z)=0, \forall \eta \in \mathcal{J}_{M}^{1}(U)\right\}, \quad \forall U^{\text {open }} \subset M .
\end{aligned}
$$

If $\mathscr{Z}$ is a distribution, it is the characteristic system of its differential ideal. The pointwise evaluation of the elements of $\mathcal{J}_{M}^{1}$ yields in this case a smooth subbundle $\boldsymbol{Z}^{0} M$ of $\mathrm{C} T^{*} M$, given by

$$
\boldsymbol{Z}^{0} M=\bigsqcup_{p \in M} \boldsymbol{Z}_{p}^{0} M, \text { with } \boldsymbol{Z}_{p}^{0} M=\left\{\eta \in \mathrm{C} T_{p}^{*} M \mid \eta(Z)=0 \forall Z \in \mathscr{Z}(M)\right\} .
$$

In general, (2.6) defines a subset of the complexified tangent bundle of $M$.

DEFINITION 2.2. Let $\mathscr{Z}$ be a generalized distribution of smooth complex vector fields on $M$ and $p_{0} \in M$. We say that $\mathscr{Z}$ is completely integrable at $p_{0}$ if

$$
\forall \eta \in \boldsymbol{Z}_{p_{0}}^{0} M \quad \exists u \in \mathcal{O}_{M}\left(p_{0}\right) \text { with } d u\left(p_{0}\right)=\eta .
$$

This means that (2.1) has at $p_{0}$ the largest number of differentially independent local solutions that is permitted by the rank of $\mathscr{Z}$.

\section{3 - The case of CR manifolds}

Let $\mathscr{Z}$ be a $C R$ structure of type $(n, k)$ on $M$. Complete integrability at $p_{0} \in M$ is equivalent to the existence of a complex $C R$-immersion of class $\mathcal{C}^{1}$ of an open neighborhood $U$ of $p_{0}$ into $\mathrm{C}^{n+k}$.

The question of the regularity of complex $C R$-immersions seems in general a rather delicate open problem (see e.g. [31]). Note that any $\mathcal{C}^{1}$ immersion is in fact $\mathcal{C}^{\infty}$-smooth when $M$ satisfies suitable pseudo-concavity assumptions (see [2]). 
For $\mathcal{C}^{\infty}$-smooth complex local $C R$-immersions we introduce a special notation.

Definition 2.3. A CR-chart on $M$ is the datum of an open subset $U$ and of $n+k$ smooth CR functions $z_{1}, \ldots, z_{n+k} \in \mathcal{O}_{M}(U) \cap \mathcal{C}^{\infty}(U)$, such that

$$
d z_{1}(p) \wedge \cdots \wedge d z_{n+k}(p) \neq 0, \forall p \in U .
$$

Clearly $\phi(p)=\left(z_{1}(p), \ldots, z_{n+k}(p)\right)$ provides in this case a $\mathcal{C}^{\infty}$-smooth $C R$-immersion of $U$ in $\mathbb{C}^{n+k}$.

The functions $z_{1}, \ldots, z_{n+k}$ of a $C R$-chart are not independent complex coordinates when $k>0$. For each point $p_{0}$ of $U$ there are indeed $k$ real valued functions $\rho_{1}, \ldots, \rho_{k}$, defined and $\mathcal{C}^{\infty}$ on an open neighborhood $G$ of $\phi\left(p_{0}\right)$ in $\mathbb{C}^{n+k}$, with $\rho_{i}\left(z_{1}, \ldots, z_{n+k}\right)=0$ on a neighborhood of $p_{0}$, and $\partial \rho_{1}\left(\phi\left(p_{0}\right)\right) \wedge \cdots \wedge \partial \rho_{k}\left(\phi\left(p_{0}\right)\right) \neq 0$.

Definition 2.4. We say that a $C R$ manifold $M$ is locally $C R$-embeddable if the open subsets $U$ of its $C R$-charts make a covering.

Locally $C R$-embeddable $C R$ manifolds can be abstractly defined as ringed spaces, using the structure sheaf $\mathcal{O}_{M}^{\infty}=\mathcal{O}_{M} \cap \mathscr{C}^{\infty}$ of the germs of its smooth $C R$ functions.

Lemma 2.5. Let $M$ be a $C R$ manifold of type $(n, k)$ and $p_{0} \in M$. Then we can find an open neighborhood $U$ of $p_{0}$ in $M$ and a new $C R$ structure on $M$ which is locally $C R$-embeddable and agrees to infinite order with the original one at $p_{0}$.

Proof. Let $\mathscr{Z}$ be the $C R$ structure on $M$. It suffices to consider smooth functions $z_{1}, \ldots, z_{v}$ which are defined on a neighborhood of $p_{0}$, satisfy $Z z_{j}=0^{\infty}$ at $p_{0}$, and have $d z_{1}\left(p_{0}\right) \wedge \cdots \wedge d z_{v}\left(p_{0}\right) \neq 0$. To prove the existence of such functions, we observe that it is always possible to find a smooth coordinate chart $\left(U, x_{1}, \ldots, x_{m}\right)$ centered at $p_{0}$ such that $\mathscr{Z}$ is generated in $U$ by vector fields of the form

$$
Z_{i}=\frac{\partial}{\partial x_{i}}+i \frac{\partial}{\partial x_{i+n}}+\sum_{j=n+1}^{m} a_{j}(x) \frac{\partial}{\partial x_{j}}, \quad \text { with } a_{j}(x)=O(|x|) .
$$

Let $L_{i}=\frac{\partial}{\partial x_{i}}+i \frac{\partial}{\partial x_{i+n}}$, and $R_{i}=\sum_{j=n+1}^{m} a_{j}(x) \frac{\partial}{\partial x_{j}}$. 
We denote by $\mathfrak{m}$ the maximal ideal of the local ring $\mathbb{C}\left\{\left\{x_{1}, \ldots, x_{m}\right\}\right\}$ of formal power series of $x_{1}, \ldots, x_{m}$. We obtain formal power series solution to (2.1) by constructing by recurrence sequences $\left\{f_{h}\right\}_{h \geq 0} \subset \mathbb{C}\left\{\left\{x_{1}, \ldots, x_{m}\right\}\right\}$ which solve the equations

$$
\begin{cases}f_{h} \in \mathfrak{m}^{h}, & \text { for } j=1, \ldots, n, \\ L_{j} f_{1} \in \mathfrak{m}, & \text { for } j=1, \ldots, n . \\ L_{j} f_{h+1}+R_{j} f_{h} \in \mathfrak{m}^{h+1},\end{cases}
$$

We observe that, taking $f_{1}$ equal to $x_{i}+i x_{i+n}$ for $i=1, \ldots, n$, or to $x_{2 n+i}$, for $i=1, \ldots, k$, we obtain $v$ independent solutions of $L_{i} f_{1}=0$ for $1 \leq i \leq n$.

Assume now that $d \geq 1$ and $f_{d} \in \mathfrak{m}^{d}$ satisfies

$$
L_{i} f_{d}+R_{i} f_{d-1} \in \mathfrak{m}^{d}, \quad \text { for } \quad 1 \leq i \leq n .
$$

The integrability conditions yield $\left[Z_{i}, Z_{j}\right]=0$ for $1 \leq i, j \leq n$. Hence we obtain

$$
0=\left[Z_{i}, Z_{j}\right] f_{d}=-L_{i} R_{j} f_{d}+L_{j} R_{i} f_{d}+\left[R_{i}, R_{j}\right] f_{d} .
$$

We have $R_{i} f_{d} \in \mathfrak{m}^{d}$, and hence there is a polynomial $g_{i, d} \in \mathrm{C}\left[x_{1}, \ldots, x_{m}\right]$, homogeneous of degree $d$, such that $R_{i} f_{d}-g_{i, d} \in \mathfrak{m}^{d+1}$. Since $\left[R_{i}, R_{j}\right] f_{d} \in \mathfrak{m}^{d+1}$, we obtain from $(* *)$ that $L_{i} g_{j, d}=L_{j} g_{i, d}$ for all $1 \leq i, j \leq n$ and therefore there is a polynomial $f_{d+1} \in \mathbb{C}\left[x_{1}, \ldots, x_{m}\right]$, homogeneous of degree $d+1$, such that $L_{i} f_{d+1}=g_{i, d}$ for $i=1, \ldots, n$. The series $\sum f_{d}$ of the terms of a sequence $\left\{f_{d}\right\}$ solving $(*)$ is a formal power series solution of (2.1).

In particular, we can find solutions $\left\{z_{1}\right\}, \ldots,\left\{z_{v}\right\} \in \mathbb{C}\left\{\left\{x_{1}, \ldots, x_{m}\right\}\right\}$ to (2.1) with $d\left\{z_{i}\right\}(0)=d x_{i}(0)+i d x_{i+n}(0)$ for $i=1, \ldots, n$ and $d\left\{z_{i}\right\}(0)=$ $d x_{n+i}(0)$ for $i=n+1, \ldots, v$. It suffices then to take smooth functions $z_{1}, \ldots, z_{v}$ having Taylor series $\left\{z_{1}\right\}, \ldots,\left\{z_{v}\right\}$ at 0 .

\section{4 - Characteristic bundle and Levi form [33]}

The underlying real distribution and the characteristic bundle of $\mathscr{Z}$ are:

(2.8) $\mathcal{H}=\{\operatorname{Re} Z \mid Z \in \mathscr{Z}\}, \quad$ i.e $\mathcal{H}(U)=\{\operatorname{Re} Z \mid Z \in \mathscr{Z}(U)\}, \forall U^{\text {open }} \subset M$, (2.9) $H^{0} M=\left\{\xi \in T^{*} M \mid \xi(X)=0, \forall X \in \mathcal{H}(M)\right\}$. 
To each characteristic covector $\xi_{0} \in H_{p_{0}}^{0} M$ we associate a Hermitian symmetric form on $\boldsymbol{Z}_{p_{0}} M$, by

$$
\mathfrak{Q}_{\xi_{0}}\left(Z_{1}, Z_{2}\right)=i \xi_{0}\left(\left[Z_{1}, \bar{Z}_{2}\right]\right), \quad \forall Z_{1}, Z_{2} \in \mathscr{Z}(M) .
$$

In fact a straightforward verification shows that the value of the right hand side of (2.10) only depends on $Z_{1}\left(p_{0}\right), Z_{2}\left(p_{0}\right) \in \boldsymbol{Z}_{p_{0}} M$.

Moreover, $\mathfrak{L}_{\xi_{0}}\left(Z_{1}, Z_{2}\right)=0$ if one of the two vector fields is real valued on a neighborhood of $p_{0}$. Thus $\mathfrak{Q}_{\xi_{0}}$ defines a Hermitian symmetric form on the quotient of $\boldsymbol{Z}_{p_{0}} M$ by the subspace $\boldsymbol{N}_{p_{0}} M=\left\{Z\left(p_{0}\right) \mid Z \in \mathscr{Z}(M) \cap \overline{\mathscr{Z}(M)}\right\}$, consisting of the values at $p_{0}$ of the complex multiples of the real vector fields in $\mathscr{Z}(M)$. Set

$$
\check{\boldsymbol{Z}}_{p_{0}} M=\boldsymbol{Z}_{p_{0}} M / \boldsymbol{N}_{p_{0}} M .
$$

If $\xi_{0} \in H_{p_{0}}^{0} M$, then (2.10) defines a Hermitian symmetric form $\boldsymbol{L}_{\xi_{0}}$ on $\check{\boldsymbol{Z}}_{p_{0}} M$, that we call the Levi form of $\mathscr{Z}$ at $\xi_{0}$.

Definition 2.6. Let $p_{0} \in M$ and $\xi_{0} \in H_{p_{0}}^{0} M$. We say that $\mathscr{Z}$ is $q$ pseudoconvex at $\xi_{0}$ if $\boldsymbol{L}_{\xi_{0}}$ is nondegenerate and has exactly $q$ positive eigenvalues on $\check{\boldsymbol{Z}}_{p_{0}} M$.

If $\mathscr{Z}$ is 1-pseudoconvex at some $\xi_{0} \in H_{p_{0}}^{0} M$, we say that $\mathscr{Z}$ is Lorentzian at $p_{0}$.

If $\mathscr{Z}(M)$ is generated by a single vector field $L$ near $p_{0}$, the condition of being Lorentzian at $p_{0}$ means that $L\left(p_{0}\right), \bar{L}\left(p_{0}\right)$, and $[L, \bar{L}]\left(p_{0}\right)$ are linearly independent in $\mathrm{CT}_{p_{0}} M$.

\section{5 - Reduction of complete integrability to the case of CR manifolds}

When $\boldsymbol{N}_{p} M$ has constant dimension on a neighborhood $U$ of $p_{0} \in M$, then the real vector fields in $\mathscr{Z}(U)$ define an involutive distribution $\mathscr{N}$ of real vector fields on $U$. By the Frobenius theorem, there is an open neighborhood $W$ of $p_{0}$ in $U$ and a smooth fibration $\pi: W \rightarrow B$ of $W$ such that $B$ is a smooth manifold and the fibers of $\pi$ are integral submanifolds of $\mathscr{N}$. One easily proves

Lemma 2.7. There is a CR structure $\mathscr{Z}^{\prime}$ on $B$ such that for every $p \in W$ we have $\mathcal{O}_{M,(p)}=\pi^{*} \mathcal{O}_{B,(\pi(p))}$, and $\mathscr{Z}$ is completely integrable at $p \in W$ if and only if $\mathscr{Z}^{\prime}$ is completely integrable at $\pi(p)$. 


\section{Involutive systems which are not completely integrable at $p_{0}$}

In this section, we give a weak generalization of the results of $\S 1$ to Lorentzian $C R$ manifolds $M$ with arbitrary $C R$-codimension $k \geq 1$ and $C R$-dimension $n \geq 2$. We recall that $m=\operatorname{dim}_{\mathbb{R}} M=2 n+k$, and we set $v=n+k$.

We closely follow the arguments of $\S 1$.

Assume that $M$ is locally $C R$-embeddable and Lorentzian at $p_{0}$. Then there is a real valued function $\rho$, defined on a full generic complex neighborhood of $p_{0}$, and vanishing on $M$, whose complex Hessian has signature $(1, n-1)$ on $H_{p_{0}} M$. By adding a real valued function $\psi$, vanishing to the second order on $M$, we obtain a new function $\rho+\psi$, vanishing on $M$, with $\partial \bar{\partial}(\rho+\psi)=\partial \bar{\partial} \rho$ on $H_{p_{0}} M$, while the signature of the full complex Hessian of $\rho+\psi$ has been arbitrarily adjusted in the transversal directions to $M$. Then there is a $C R$-chart $\left(U, z_{1}, \ldots, z_{v}\right)$ centered at $p_{0}$, with $d z^{i}\left(p_{0}\right)$ real for $i=n+1, \ldots, v$, and

$$
\operatorname{Im} z_{v}+z_{v} \bar{z}_{v}+\sum_{i=1}^{n-1} z_{j} \bar{z}_{j}=\sum_{i=n}^{v-1} z_{j} \bar{z}_{j}+O\left(|z|^{3}\right) \quad \text { on } U .
$$

By shrinking, we get $\sum_{i=n}^{v-1} z_{i} \bar{z}_{i} \geq \frac{1}{2} \operatorname{Im} z_{v}$ on $U$.

We consider the map $\pi: U \ni p \rightarrow w=\left(z_{1}(p), \ldots, z_{n-1}(p), z_{v}(p)\right) \in \mathbb{C}^{n}$. By a further shrinking, we can assume that there is an open ball $B \subset \mathrm{C}^{n}$, centered at 0 , such that

- $\pi(U)=\bar{\omega}$, with $B \backslash \omega$ strictly convex, and $\partial \omega \cap B$ smooth;

- if $\operatorname{Im} \tau \geq 0$, then $\left\{w \in B \mid \operatorname{Im} w_{n}=\tau\right\} \subset \omega$;

- for all $w \in \omega$ the set $M_{w}=\pi^{-1}(w)$ is diffeomorphic to the sphere $S^{k}$;

- for $w \in \partial \omega \cap B$ the set $M_{w}=\pi^{-1}(w)$ is a point.

As in $\S 1$, we have:

LEMma 3.1. If $u \in \mathcal{O}_{M}(U)$, then

$$
F(w)=\int_{M_{w}} u d z_{n} \wedge \cdots \wedge d z_{v-1}=0, \quad \forall w \in \omega .
$$

Proof. We prove first that $F$ is holomorphic on $\omega$.

Fix any polycylinder $D=D_{1} \times \cdots \times D_{n}$ in $\omega$, with $D_{i}=$ $\left\{\tau \in \mathbb{C}|| \tau-\tau_{i} \mid \leq \varepsilon_{i}\right\}$. For $1 \leq j \leq n$ we set $\partial_{j}(D)=\left\{w \in D|| w_{j}-\tau_{j} \mid=\varepsilon_{j}\right\}$, 
$\left.\gamma_{j}=\frac{\partial}{\partial \bar{w}_{j}}\right\rfloor\left(d \bar{w}_{1} \wedge \cdots \wedge d \bar{w}_{n}\right)$ and consider the integral

$$
\oint_{\partial_{j} D} F(w) d w_{1} \wedge \cdots \wedge d w_{n} \wedge \gamma_{j}=\oint_{\partial_{j} D} d w_{1} \wedge \cdots \wedge d w_{n} \wedge \gamma_{j} \int_{M_{w}} u d z_{n} \wedge \cdots \wedge d z_{v-1} .
$$

Let $N_{i}=\pi^{-1}\left(\partial_{i} D\right)$ and $N=\pi^{-1}(D)$. We have $\partial N=\sum_{i=1}^{n} \pm N_{i}$. Moreover, the form $u d z_{1} \wedge \cdots \wedge d z_{v} \wedge \pi^{*} \gamma_{j}$ is zero on $N_{i}$ for $i \neq j$. Thus we obtain:

$$
\begin{aligned}
& \oint_{\partial_{j} D} F(w) d w_{1} \wedge \cdots \wedge d w_{n} \wedge \gamma_{j}= \pm \int_{N_{j}} u d z_{1} \wedge \cdots \wedge d z_{v} \wedge \pi^{*} \gamma_{j} \\
& \quad=\sum_{i=1}^{n} \int_{N_{i}} \pm u d z_{1} \wedge \cdots d z_{v} \wedge \pi^{*} \gamma_{j}= \pm \int_{N} d u \wedge d z_{1} \wedge \cdots d z_{v} \wedge \pi^{*} \gamma_{j}=0
\end{aligned}
$$

because $d u \in\left\langle d z_{1}, \ldots, d z_{v}\right\rangle$ by the assumption that $u \in \mathcal{O}_{M}(U)$. This equality, valid for all closed polycylinder $D$ in $\omega$ and all $1 \leq j \leq n$, implies that $F$ is holomorphic in $\omega$. Clearly $F(w) \rightarrow 0$ when $w \rightarrow \partial \omega \cap B$, because $M_{w_{0}}$ is a point for $w_{0} \in \partial \omega \cap B$, and hence $F=0$ on $\omega$ by Holmgren's uniqueness theorem, since $\bar{\partial}$ has constant coefficients in $\mathbb{C}^{n}$.

Let $\psi$ be a smooth function with compact support in $\mathbb{C}$, and set

Then $\frac{\partial \hat{\psi}}{\partial \bar{\tau}}=\psi$ and therefore

$$
\hat{\psi}(\tau)=\frac{1}{2 \pi i} \iint \frac{\psi(\zeta) d \zeta \wedge d \bar{\zeta}}{\zeta-\tau} .
$$

$$
\psi^{\sharp}(\zeta, \tau)=\bar{\zeta} \psi(\tau) d \bar{\tau}+\hat{\psi}(\tau) d \bar{\zeta}=\bar{\partial}(\bar{\zeta} \hat{\psi}(\tau))
$$

is a $\bar{\partial}$-closed form in $\mathbb{C}^{2}$, with

$$
d \psi^{\sharp}=\frac{\partial \hat{\psi}(\tau)}{\partial \tau} d \tau \wedge d \bar{\zeta}+\bar{\zeta} \frac{\partial \psi(\tau)}{\partial \tau} d \tau \wedge d \bar{\tau}=d \tau \wedge \frac{\partial}{\partial \tau} \psi^{\sharp} .
$$

LEMMA 3.2. Let $U^{\prime} \Subset U$. If $\psi_{i}$, for $i=1, \ldots, v-1$, are smooth functions of a complex variable $\tau$, with $\left|\psi_{i}\right|$ sufficiently small. Then

$$
\theta_{1}=d z_{1}+\psi_{1}^{\sharp}\left(z_{n}, z_{v}\right), \ldots, \theta_{v-1}=d z_{v-1}+\psi_{v-1}^{\sharp}\left(z_{n}, z_{v}\right), \theta_{v}=d z_{v}
$$

generate the involutive ideal sheaf $\mathcal{J}_{M}^{\prime}$ of a $C R$ structure of type $(n, k)$ on $U^{\prime}$.

Proof. The ideal sheaf is generated on $U$ by $d z_{1}, \ldots, d z_{v}$. After shrinking, we can assume that $d z_{1}, \ldots, d z_{n}, d \bar{z}_{1}, \ldots, d \bar{z}_{n}$ are linearly independent on $U$. 
Thus, by taking $\left|\psi_{i}\right|$ sufficiently small, we may keep $\theta_{1}, \ldots, \theta_{v}, \bar{\theta}_{1}, \ldots, \bar{\theta}_{n}$ linearly independent in any neighborhood $U^{\prime}$ of $p_{0}$ with $U^{\prime} \Subset U$. Moreover, since $d \psi_{i}^{\sharp}\left(z_{n}, z_{v}\right) \wedge d z_{v}=0$, for $1 \leq i<v$, we obtain $\left(d \theta_{i}\right) \wedge \theta_{1} \wedge \cdots \wedge \theta_{v}=d \psi_{i}^{\sharp}\left(z_{n}, z_{v}\right) \wedge \theta_{1} \wedge \cdots \wedge \theta_{v-1} \wedge d z_{v}=0, \forall i=1, \ldots, v-1$. This shows that the ideal sheaf $\mathcal{J}_{M}^{\prime}$ generated by $\theta_{1}, \ldots, \theta_{v}$ is involutive and defines a $C R$ structure of type $(n, k)$ on $U^{\prime}$.

Let us fix a sequence of distinct complex numbers $\left\{\tau_{j}\right\}$, such that

$$
\operatorname{Im} \tau_{j}>0 \text { for all } j, \quad \tau_{j} \rightarrow 0, \quad\left\{w_{n}=\tau_{j}\right\} \cap \omega \neq \emptyset \text { for all } j .
$$

For each $j$ we choose an open disk $\Delta_{j}$ in $\mathrm{C}$, centered at $\tau_{j}$, and such that $\bar{\Delta}_{j} \cap \bigcup_{i \neq j} \bar{\Delta}_{i}=\emptyset$. Provided the $\tau_{j}$ 's are sufficiently close to 0 , for each $j$ we can fix a point $w^{(j)} \in \omega$, with $w_{n}^{(j)}=\tau_{j}$, and $w^{(j)} \rightarrow 0$, and take the functions $\psi_{j}$ in Lemma 3.2 in such a way that

$$
\begin{gathered}
\operatorname{supp} \psi_{i}=\bigcup_{j=0}^{\infty} \bar{\Delta}_{i+j v}, \quad \text { for } i=1, \ldots, n, \\
c_{i+j v}=\int_{A_{i+j v}} \psi_{i}^{\sharp}\left(z_{n}, z_{v}\right) \wedge d z_{n} \wedge \cdots \wedge d z_{v-1} \wedge d z_{v} \text { is real and }>0,
\end{gathered}
$$

where $\left(e_{1}, \ldots, e_{n}\right.$ is the canonical basis of $\left.\mathrm{C}^{n}\right)$

$$
A_{j}=\pi^{-1}\left(\left\{w^{(j)}+\left(\tau-\tau_{j}\right) e_{n} \mid \tau \in \Delta_{j}\right\}\right) .
$$

Let $u$ be a $C R$ function on an open neighborhood $V$ of $p_{0}$ in $U$ for the structure defined by (3.3). This means that $d u_{(p)} \in \mathcal{J}_{M(p)}^{\prime}$ for all $p \in V$. Since $\mathcal{J}_{M}$ and $\mathcal{J}_{M}^{\prime}$ agree to infinite order outside $\bigcup_{j} \pi^{-1}\left(\left\{w \mid w_{n} \in \Delta_{j}\right\}\right.$, and $\bigcup_{j}\left\{w \in \omega \mid w_{n} \in \Delta_{j}\right\}$ does not disconnect $\omega$, by the argument of Lemma 3.1 we have (4.2) for all $w$ in the complement in $\pi(V) \backslash \bigcup_{j}\left\{w \in \omega \mid w_{n} \in \Delta_{j}\right\}$. Thus we obtain

$$
\begin{aligned}
0= \pm \oint_{\tau \in \partial \Delta_{j}} d \tau \int_{M_{w^{(j)+\tau e n}}} u d z_{n} \wedge \cdots \wedge d z_{v-1} & = \pm \int_{\partial A_{j}} u d z_{n} \wedge \cdots \wedge d z_{v} \\
& = \pm \int_{A_{j}} d u \wedge d z_{n} \wedge \cdots \wedge d z_{v}
\end{aligned}
$$


This yields

$$
\int_{A_{i}+j v} \frac{\partial u}{\partial z_{i}} \psi_{i}^{\sharp} \wedge d z_{n} \wedge \cdots \wedge d z_{v}=0,
$$

where, to compute $\frac{\partial u}{\partial z_{i}}$, we consider any $\mathcal{C}^{1}$-extension of $u$ as a function of the complex variables $z_{1}, \ldots, z_{v}$ for which $\bar{\partial} u=0$ at all points of $U$. Taking the limit, we observe that

$c_{i+j v}^{-1} \int_{A_{i}+j v} \frac{\partial u}{\partial z_{i}} \psi_{i}^{\sharp} \wedge d z_{n} \wedge \cdots \wedge d z_{v} \longrightarrow \frac{\partial u\left(p_{0}\right)}{\partial z_{i}} \Longrightarrow \frac{\partial u\left(p_{0}\right)}{\partial z_{i}}=0 \quad \forall i=1, \ldots, v-1$,

which, together with (2.1) shows that $d u\left(p_{0}\right) \in \mathbb{C} d z_{v}\left(p_{0}\right)$.

We have proved:

THEOREM 3.3. Let $M$ be a $C R$ manifold of type $(n, k)$ and assume that $M$ is Lorentzian at a point $p_{0}$. Then we can find a new $C R$ structure of type $(n, k)$ on a neighborhood $U$ of $p_{0}$, which agrees with the original one to infinite order at $p_{0}$, and a real codirection $\eta_{0} \in T_{p_{0}}^{*} M$ such that, if $\mathscr{Z}$ is the distribution of $(0,1)$-vector fields for this new structure, all solutions $u \in \mathcal{C}^{1}$ on a neighborhood of $p_{0}$ to the homogeneous system (2.1) satisfy $d u\left(p_{0}\right) \in \mathrm{C} \eta_{0}$.

Proof. Indeed, using Lemma 2.5 we can always reduce to the case in which $M$ is locally embeddable at $p_{0}$.

CoRollary 3.4. We can find a new CR structure of type $(n, k)$ on $U$, which agrees with the original one to infinite order at $p_{0}$, and which is not CR-embeddable at $p_{0}$.

\section{Involutive systems whose solutions are critical at $p_{0}$}

In this section we improve the result of the previous section in the case of a Lorentzian $C R$ manifold of the hypersurface type.

A proof of Theorem 4.3 is contained in [24], where, however, the result is not correctly stated (see Remark 4.4 below). However, our proof here is different and outlines, in the simpler case of the hypersurface-type, the argument used later for higher $C R$ codimension. 
We assume that $M$ has $C R$-dimension $n \geq 2$ and $C R$-codimension 1, and is Lorentzian and locally embeddable at $p_{0} \in M$. We have $m=\operatorname{dim}_{\mathbb{R}} M=n+2$ and we set $v=n+1$,

We can fix a $C R$-chart $\left(U, z_{1}, \ldots, z_{v}\right)$ centered at $p_{0}$, with

$$
\operatorname{Im} z_{v}+\sum_{i=2}^{v} z_{i} \bar{z}_{i}=z_{1} \bar{z}_{1}+O\left(|z|^{3}\right) \quad \text { on } U .
$$

By shrinking, we get that $z_{1} \bar{z}_{1} \geq \frac{1}{2} \operatorname{Im} z_{v}$ on $U$. Consider the map $\pi: U \ni p \rightarrow w=\left(z_{2}(p), \ldots, z_{v}(p)\right) \in \mathbb{C}^{n}$. By a further shrinking, we can assume that there is an open ball $B \subset \mathbb{C}^{n}$, centered at 0 , such that

- $\pi(U)=\bar{\omega}$, with $B \backslash \omega$ strictly convex, and $\partial \omega \cap B$ smooth;

- if $\operatorname{Im} \tau \geq 0$, then $\left\{w \in B \mid \operatorname{Im} w_{n}=\tau\right\} \subset \omega$;

- for all $w \in \omega$ the set $M_{w}=\pi^{-1}(w)$ is diffeomorphic to the circle $S^{1}$;

- for $w \in \partial \omega \cap B$ the set $M_{w}=\pi^{-1}(w)$ is a point.

By repeating the proof of Lemma 3.1, we obtain

Lemma 4.1. If $u \in \mathcal{O}_{M}(U)$, then

$$
F(w)=\oint_{M_{w}} u d z_{1}=0, \quad \forall w \in \omega .
$$

Since $2 z_{1} \bar{z}_{1} \geq \operatorname{Im} z_{v}$ on $U$, for any smooth function $\psi$ of a complex variable $\tau$, with $\operatorname{supp} \psi \subset\{\operatorname{Im} \tau \geq 0\}$, the function $z_{1}^{-1} \psi\left(z_{v}\right)$ can be extended to a smooth function on $U$, vanishing to infinite order on $\left\{z_{1}=0\right\} \cap U$.

LEMma 4.2. If $\psi_{i}$, for $i=1, \ldots, v$ are smooth fuctions of a complex variable $\tau$, with support contained in $\{\operatorname{Im} \tau \geq 0\}$, then

$$
\theta_{1}=d z_{1}+z_{1}^{-1} \psi_{1}\left(z_{v}\right) d \bar{z}_{v}, \ldots, \theta_{v}=d z_{v}+z_{1}^{-1} \psi_{v}\left(z_{v}\right) d \bar{z}_{v}
$$

(the functions $z_{1}^{-1} \psi_{i}\left(z_{v}\right)$ are put $=0$ for $z_{1}=0$ ) generate the ideal sheaf $\mathcal{J}_{U^{\prime}}^{\prime}$ of a $C R$ structure of type $(n, 1)$ in a neighborhood $U^{\prime}$ of $p_{0}$ in $U$, which agrees to infinite order with the original one at $p_{0}$.

Proof. By the condition on the supports, the functions $z_{1}^{-1} \psi_{i}\left(z_{v}\right)$ are smooth on $U$ and vanish to infinite for $z_{1}=0$, and in particular at $p_{0}$. Thus $\theta_{1}, \ldots, \theta_{v}, \bar{\theta}_{1}, \ldots, \bar{\theta}_{n}$ yield a basis of $\mathrm{C} T_{p} M$ for $p$ in a suitable neighborhood $U^{\prime}$ of $p_{0}$, and agree with $d z_{1}, \ldots, d z_{v}, d \bar{z}_{1}, \ldots, d \bar{z}_{n}$ to infinite order at $p_{0}$. 
We have moreover

$$
d \theta_{i}=z_{1}^{-1} \frac{\partial \psi_{i}\left(z_{v}\right)}{\partial z_{v}} d z_{v} \wedge d \bar{z}_{v}-z_{1}^{-2} \psi_{i}\left(z_{v}\right) d z_{1} \wedge d \bar{z}_{v} .
$$

Hence

$$
d \theta_{i} \wedge \theta_{1} \wedge \cdots \wedge \theta_{v}=d \theta_{i} \wedge d z_{1} \wedge \cdots \wedge d z_{v}=0
$$

shows that $\mathcal{J}_{U^{\prime}}^{\prime}$ is involutive. The proof is complete.

Let us fix a sequence of distinct complex numbers $\left\{\tau_{j}\right\}$, such that

$$
\operatorname{Im} \tau_{j}>0 \text { for all } j, \quad \tau_{j} \rightarrow 0, \quad\left\{w_{n}=\tau_{j}\right\} \cap \omega \neq \emptyset \text { for all } j .
$$

For each $j$ we choose an open disk $\Delta_{j}$ in $\mathrm{C}$, centered at $\tau_{j}$, and such that $\bar{\Delta}_{j} \cap \bigcup_{i \neq j} \bar{\Delta}_{i}=\emptyset$. Provided the $\tau_{j}$ 's are sufficiently close to 0 , for each $j$ we can fix a point $w^{(j)} \in \omega$, with $w_{n}^{(j)}=\tau_{j}$, and $w^{(j)} \rightarrow 0$, and take the functions $\psi_{j}$ in Lemma 4.2 in such a way that

$$
\begin{gathered}
\operatorname{supp} \psi_{i}=\bigcup_{j=0}^{\infty} \bar{\Delta}_{i+j(v+1)}, \text { for } i=1, \ldots, v, \\
c_{i+j(v+1)}=\int_{A_{i+j(v+1)}} z_{1}^{-1} \psi_{i}\left(z_{v}\right) d \bar{z}_{v} \wedge d z_{1} \wedge d z_{v} \text { is real and }>0,
\end{gathered}
$$

where

$$
A_{j}=\pi^{-1}\left(\left\{w^{(j)}+\left(\tau-\tau_{j}\right) e_{n} \mid \tau \in \Delta_{j}\right\}\right) .
$$

Here we denoted by $e_{1}, \ldots, e_{n}$ the canonical basis of $\mathrm{C}^{n}$.

Let $u$ be a $C R$ function on an open neighborhood $V$ of $p_{0}$ in $U^{\prime}$ for the structure defined by (5.2). This means that $d u_{(p)} \in \mathcal{J}_{U^{\prime}(p)}^{\prime}$ for all $p \in V$. Since $\mathcal{J}_{M}$ and $\mathcal{J}_{U^{\prime}}^{\prime}$ agree to infinite order on $\pi\left(U^{\prime}\right)$ outside $\bigcup_{i} \operatorname{supp} \psi_{i}(w)$, and this set does not disconnect $U$, by the argument of Lemma 3.1 we have (4.2) for all $w$ in the complement in $\pi(V)$ of $\bigcup_{j}\left\{w \in \omega \mid w_{n} \in \Delta_{j}\right\}$. Thus we obtain

$$
0= \pm \oint_{\tau \in \partial \Delta_{j}} d \tau \oint_{M_{w^{(j)}+e_{n}}} u d z_{n}= \pm \int_{\partial A_{j}} u d z_{1} \wedge d z_{v}= \pm \int_{A_{j}} d u \wedge d z_{1} \wedge d z_{v} .
$$

This yields

$$
I_{i+j(v+1)}(u)=\int_{A_{i}+j(v+1)} \frac{\partial u}{\partial z_{i}} z_{1}^{-1} \wedge d \bar{z}_{v} \wedge d z_{1} \wedge d z_{v}=0,
$$


where, to compute $\frac{\partial u}{\partial z_{i}}$, we consider any $\mathcal{C}^{1}$-extension of $u$ as a function of the complex variables $z_{1}, \ldots, z_{v}$ for which $\bar{\partial} u=0$ at all points of $V$. When $j \rightarrow \infty, \quad c_{i+j(v+1)}^{-1} I_{i+j(v+1)} \rightarrow \frac{\partial u\left(p_{0}\right)}{\partial z_{i}}$. Hence, from (4.4) we obtain that $\frac{\partial u\left(p_{0}\right)}{\partial z_{i}}=0$ for $1 \leq i \leq v$, which, together with (2.1) shows that $d u\left(p_{0}\right)=0$.

We have proved:

THEOREM 4.3. If $M$ is a CR manifold of type $(n, 1)$ and is Lorentzian at $p_{0} \in M$, then we can find a new $C R$ structure of type $(n, 1)$ on an open neighborhood $U$ of $p_{0}$ in $M$, which agrees with the original one to infinite order at $p_{0}$, such that, if $\mathscr{Z}$ is the distribution of $(0,1)$-vector fields for this new structure, all solutions $u \in \mathcal{C}^{1}$ on a neighborhood of $p_{0}$ to the homogeneous system (2.1) satisfy $d u\left(p_{0}\right)=0$.

Proof. We can apply the discussion above after reducing, by Lemma 2.5 , to the case in which $M$ is locally $C R$-embeddable at $p_{0}$.

REMARK 4.4. The statement of Theorem 4.3 is weaker than that of Theorems I, II in [24], in which the perturbed $C R$ structure is claimed to exist globally. However, the final part of the argument there has a serious gap. Let us explain in what it consists. Let $L_{1}, \ldots, L_{n}$ and $\Lambda_{1}, \ldots, \Lambda_{n}$ be basis for the original and the perturbed structure, respectively, defined on a coordinate neighborhood $(U, x)$, centered at a chosen point $p \in M$. In [24, p. 290, (4.10)] new global structures are defined, which agree with the original one outside of $U$, and having, in $U$, the basis $L_{j}^{(v)}=g_{v} \Lambda_{j}+\left(1-g_{v}\right) L_{j}, j=1, \ldots, n$. Here $g_{v}(x)=g\left(x / r_{v}\right)$ for a scalar function $g$, having a star-shaped compact support in $x(U)$, equal to 1 on a neighborhood of 0 , and $0<r_{v}<1, r_{v} \searrow 0$. The flaw consists in the fact that these new structures, which are perturbations with arbitrarily small compact support of the given one, fail to be formally integrable in $0<g_{v}<1$.

We think instead that, when $n>1$, there are geometrical obstructions to the existence of perturbations with arbitrarily small compact supports and that in fact propagation should be a foremost important topic in further investigations on the deformations of $C R$ structures. 


\section{The case of higher codimension}

In this section we extend the result of Theorem 4.3 to some $C R$ manifolds with $C R$ dimension and $C R$ codimension both greater than 1 . To this aim we will first recall some results on weak unique continuation and next consider morphisms of $C R$ manifolds.

5.1 - Minimal locally CR-embeddable CR manifolds and unique continuation

We recall that a $C R$ submanifold $M$ is minimal at $p_{0} \in M$ if there is no germ $\left(N, p_{0}\right)$ of $C R$ submanifold of $M$ at $p_{0}$, having the same $C R$ dimension, but smaller $C R$ codimension. We have

LemMa 5.1. Assume that $M$ is minimal and locally CR-embeddable at $p_{0} \in M$.

Let $\left(S, p_{0}\right)$ be a germ of a $C R$ submanifold of $M$, of type $(0, v)$. Then a germ $f \in \mathcal{O}_{M,\left(p_{0}\right)}$ of a $C R$ function at $p_{0}$, vanishing on $\left(S, p_{0}\right)$, is equal to 0 .

If $M$ is minimal and locally $C R$-embeddable at all points, then the $C R$ functions on $M$ satisfy the weak unique continuation principle.

Proof. In the first part of the proof, we can assume that $M$ is a generic $C R$ submanifold of an open set in $\mathrm{C}^{v}$. For any open neighborhood $U$ of $p_{0}$ in $M$, there are an open neighborhood $U_{0}$ of $p_{0}$ in $U$, and an open wedge $W$ in $\mathrm{C}^{v}$, with edge $U_{0}$, such that, the restriction $\left.u\right|_{U_{0}}$ of any $u \in \mathcal{O}_{M}(U)$ is the boundary value of a holomorphic function $\tilde{u}$, defined on $W$ (see $[43,44,6]$ ). Assume now that $u \in \mathcal{O}_{M}(U)$ vanishes on $S$. Then $\tilde{u}=0$ by the edge of the wedge theorem (see [39]), and therefore $u=0$.

The last statement follows by unique continuation for holomorphic functions on open subsets of $\mathrm{C}^{v}$.

\section{2 - CR-maps with simple singularities}

Let $M, N$ be $C R$ manifolds. A smooth map $\pi: M \rightarrow N$ is $C R$ if $d \pi\left(T^{0,1} M\right) \subset T^{0,1} N$. We say that $\pi$ is

- a $C R$-immersion if ker $d \pi=0$ and $d \pi\left(T^{0,1} M\right)=d \pi(\mathrm{C} T M) \cap T^{0,1} N$;

- a $C R$-submersion if $d \pi\left(T_{p} M\right)=T_{\pi(p)} N$ and $d \pi\left(T_{p}^{0,1} M\right)=T_{\pi(p)}^{0,1} N$, $\forall p \in M$; 
- a local $C R$-diffeomorphism if it is at the same time a $C R$-immersion and a $C R$-submersion.

Next we consider critical points of some $C R$-maps.

Let $k \geq 1$ and $\pi: M \rightarrow N$ a $C R$-map, with $M$ of type $(n, k)$ and $N$ of type $(n, k-1)$.

If $p_{0} \in M$ is not a critical point of $\pi$, then $\pi$ is a $C R$-submersion near $p_{0}$.

Assume now that $p_{0}$ is a critical point of $\pi$, and $q_{0}=\pi\left(p_{0}\right)$ the corresponding critical value. Then the rank of $d \pi\left(p_{0}\right)$ is less than $2 n+k-1$. Assume that it is exactly equal to $2 n+k-2$. Then the dual map $d \pi^{*}\left(p_{0}\right): T_{q_{0}}^{*} N \rightarrow T_{p_{0}}^{*} M$ is not injective, and has a 1-dimensional kernel.

Definition 5.2. If $\operatorname{ker} d \pi^{*}\left(p_{0}\right) \cap H_{q_{0}}^{0} N=\{0\}$, we say that $\pi$ has at $p_{0}$ a $C R$-noncharacteristic singularity.

Assume that this is the case and fix $0 \neq \eta_{0} \in \operatorname{ker} d \pi^{*}\left(p_{0}\right)$. Then there is $\eta_{0}^{\prime}$, uniquely determined modulo $H_{q_{0}}^{0} N$, such that $\eta_{0}+i \eta_{0}^{\prime} \in \boldsymbol{Z}_{q_{0}}^{0} N$, and we obtain an element $\xi_{0} \in H_{p_{0}}^{0} M$, with $0 \neq \xi_{0}=d \pi^{*}\left(p_{0}\right)\left(\eta_{0}^{\prime}\right)$.

DEFINITION 5.3. If we can choose $\eta_{0}^{\prime}$ in such a way that $\boldsymbol{L}_{\xi_{0}}$ has 1 positive and $n-1$ negative eigenvalues, we say that $\pi$ has a Lorentzian $C R$-non characteristic singularity at $p_{0}$.

Assume now that $M$ and $N$ are locally $C R$-embeddable at $p_{0}, q_{0}$, respectively, and that $\boldsymbol{L}_{\xi_{0}}$ has 1 positive and $(n-1)$ negative eigenvalues. We set $v=n+k$. We can choose $C R$-charts $\left(U ; z_{1}, \ldots, z_{v}\right)$ of $M$, centered at $p_{0}$, and $\left(W ; w_{2}, \ldots, w_{v}\right)$ of $N$, centered at $q_{0}$, with $\pi(U) \subset W$ and $z_{j}=\pi^{*} w_{j}$ for $j=2, \ldots, v$, such that $i \xi_{0}=d z_{v}\left(p_{0}\right)$, and

$$
\operatorname{Im} z_{v}=h(z) \quad \text { on } U, \text { with } h(z)=z_{1} \bar{z}_{1}-\sum_{i=2}^{v} z_{i} \bar{z}_{i}+O\left(|z|^{3}\right) .
$$

Lemma 5.4. Let $D=\left\{p \in U \mid z_{1}(p)=0\right\}$. Then, there is an open neighborhood $U^{\prime}$ of $p_{0}$ in $U$, an open neighborhood $\omega$ of $q_{0}$ in $N$, and an open domain $\omega_{-}$in $\omega$, with $q_{0} \in \partial \omega_{-}$, such that

(1) $\omega_{-} \subset \pi\left(U^{\prime}\right) \subset \omega, \pi\left(D \cap U^{\prime}\right) \subset \partial \omega$ and $\omega$ is strictly pseudoconcave at $q_{0}$

(2) $\pi: U^{\prime} \rightarrow N$ is proper and, for $q \in \pi\left(U^{\prime}\right), \pi^{-1}(q)$ is either a point or $i s$ diffeomorphic to a circle.

Proof. Provided $U$ is sufficiently small, the restriction of $\pi$ to $D$ is a smooth diffeomorphism of $D$ onto a closed hypersurface $\pi(D)$ in an 
open neighborhood $\omega$ of $q_{0}$ in $N$. By further shrinking, we can assume that $A \backslash \pi(D)$ consists of two connected components $\omega_{+}$and $\omega_{-}$and that $\omega_{-} \subset \pi(U)$.

Since $\omega_{-}=\left\{\operatorname{Im} w_{v}+\sum_{i=2}^{v} w_{i} \bar{w}_{i}+O\left(|w|^{3}\right)>0\right\}$ near $q_{0}$, we have $q_{0} \in \partial \omega_{-}$ and $\omega_{-}$strictly pseudoconcave at $q_{0}$. Moreover, by taking $U$ small, we can assume that

$$
\operatorname{Im} z_{v}+\frac{3}{2} \sum_{i=2}^{v} z_{i} \bar{z}_{i} \geq \frac{1}{2} z_{1} \bar{z}_{1} \quad \text { on } U
$$

and therefore we obtain an $U^{\prime}$ satisfying (1) and (2) by setting $U^{\prime}=U \cap \pi^{-1}(\omega)$ for a smaller neighborhood $\omega$ of $q_{0}$ in $N$.

\section{3 - Perturbation of the $C R$ structure of $M$}

We keep the notation of $\S 5.2$, and we shall assume that (1) and (2) of Lemma 5.4 hold true with $U^{\prime}=U$.

Lemma 5.5. Assume that $N$ is a minimal $C R$ manifold. If $u$ is a $C R$ function on a connected open neighborhood $V$ of $p_{0}$ in $U$, then

$$
g(q):=\oint_{\pi^{-1}(q)} u d z_{1}=0, \quad \forall q \in \pi(V) .
$$

Proof. First we note that $W=\pi(V) \cup(\omega \backslash \pi(U))$ is a neighborhood of $q_{0}$ in $N$. The function $g$, equal to the left hand side of (5.1) for $w \in \pi(V)$ and 0 on $W \backslash \pi(V)$ is continuous, because the fiber $\pi^{-1}(q)$ shrinks to a point when $q \rightarrow \partial \pi(V) \cap \omega$. Since $\pi(V)$ is connected and its connected component in $W$ contains an open subset where $g=0$, our contents follows by the weak unique continuation principle (see Lemma 5.1) if we show that $g$ is a $C R$ function on $W$. To this aim, it suffices to show that

$$
\int_{N} d g \wedge \eta=0, \quad \forall \eta \in \Omega_{0}^{2 n+k-2}(W) \cap \mathcal{J}_{N}^{n+k-1}(W),
$$

where $\Omega_{0}^{*}(W)$ means smooth exterior forms with compact support in $W$. We note that $\pi^{*} \eta \in \Omega_{0}^{2 n+k-2}(V) \cap \mathcal{J}_{M}^{n+k-1}(W)$, because the map $\pi$ is $C R$ and proper. Thus we obtain

$$
\int_{N} d g \wedge \eta=\int_{M} d u \wedge d z_{1} \wedge \pi^{*} \eta=0,
$$


because $u$ is $C R$ on a neighborhood of the support of $d z_{1} \wedge \pi^{*} \eta \in$ $\Omega_{0}^{2 n+k-2}(V) \cap \mathcal{J}_{M}^{n+k}(V)$. The proof is complete.

By shrinking, we get $2 z_{1} \bar{z}_{1} \geq \operatorname{Im} z_{v}$ on $U$. In particular, if $\psi$ is a smooth function of one complex variable $\tau$, with $\operatorname{supp} \psi \subset\{\operatorname{Im} \tau \geq 0\}$, the function $z_{1}^{-1} \psi\left(z_{v}\right)$ can be extended to a smooth function on $U$, vanishing to infinite order on $\left\{z_{1}=0\right\} \cap U$.

LEMMA 5.6. If $\psi_{i}$, for $i=1, \ldots, v$ are smooth fuctions of a complex variable $\tau$, with support contained in $\{\operatorname{Im} \tau \geq 0\}$, then

$$
\theta_{1}=d z_{1}+z_{1}^{-1} \psi_{1}\left(z_{v}\right) d \overline{z_{v}}, \ldots, \theta_{v}=d z_{v}+z_{1}^{-1} \psi_{v}\left(z_{v}\right) d \overline{z_{v}}
$$

(the functions $z_{1}^{-1} \psi_{i}\left(z_{v}\right)$ are put $=0$ for $z_{1}=0$ ) generate the ideal sheaf $\mathcal{J}_{U^{\prime}}^{\prime}$ of a $C R$ structure of type $(n, k)$ in a neighborhood $U^{\prime}$ of $p_{0}$ in $U$, which agree to infinite order with the original one at $p_{0}$.

Proof. By the condition on the supports, the functions $z_{1}^{-1} \psi_{i}\left(z_{v}\right)$ are smooth on $U$ and vanishing to infinite for $z_{1}=0$, and in particular at $p_{0}$. Thus $\theta_{1}, \ldots, \theta_{v}, \bar{\theta}_{1}, \ldots, \bar{\theta}_{n}$ yield a basis of $\mathrm{C} T_{p} M$ for $p$ in a suitable neighborhood $U^{\prime}$ of $p_{0}$, and agree with $d z_{1}, \ldots, d z_{v}, d \bar{z}_{1}, \ldots, d \bar{z}_{n}$ to infinite order at $p_{0}$. We have moreover

$$
d \theta_{i}=z_{1}^{-1} \frac{\partial \psi_{i}\left(z_{v}\right)}{\partial z_{v}} d z_{v} \wedge d \bar{z}_{v}-z_{1}^{-2} \psi_{i}\left(z_{v}\right) d z_{1} \wedge d \bar{z}_{v} .
$$

Hence

$$
d \theta_{i} \wedge \theta_{1} \wedge \cdots \wedge \theta_{v}=d \theta_{i} \wedge d z_{1} \wedge \cdots \wedge d z_{v}=0
$$

shows that $\mathcal{J}_{U^{\prime}}^{\prime}$ is involutive. The proof is complete.

Let us fix a sequence of distinct complex numbers $\left\{\tau_{j}\right\}$, such that

$$
\operatorname{Im} \tau_{j}>0 \text { for all } j, \quad \tau_{j} \rightarrow 0, \quad\left\{w_{n}=\tau_{j}\right\} \cap \omega \neq \emptyset \text { for all } j .
$$

For each $j$ we choose an open disk $\Delta_{j}$ in $\mathrm{C}$, centered at $\tau_{j}$, in such a way that $\bar{\Delta}_{j} \cap \bigcup_{i \neq j} \bar{\Delta}_{i}=\emptyset$. Next we choose balls $B_{j}$ in $\mathbb{C}^{v-2}$ with

$$
K_{j}=\left\{q \in \omega \mid\left(w_{2}(q), \ldots, w_{v-1}(q)\right) \in B_{j}, w_{v} \in \bar{\Delta}_{j}\right\} \Subset \omega .
$$

We set $\partial_{0} K_{j}=\left\{q \in K_{j} \mid w_{v}(q) \in \partial \Delta_{j}\right\}, A_{j}=\pi^{-1}\left(K_{j}\right)$. Note that the $A_{j}$ 's are compact because $\pi$ is proper. 
Then we take the functions $\psi_{i}$ in Lemma 5.6 in such a way that, for suitable forms $\eta_{j} \in \Omega_{0}^{n-1}\left(B_{j}\right)$, we have

$$
\begin{gathered}
\operatorname{supp} \psi_{i}=\bigcup_{j=0}^{\infty} \bar{\Delta}_{i+j(v+1)}, \quad \text { for } \quad i=1, \ldots, v, \\
c_{i+j(v+1)}=\int_{A_{i+j(v+1)}} z_{1}^{-1} \psi_{i}\left(z_{v}\right) d \bar{z}_{v} \wedge d z_{1} \wedge \cdots \wedge d z_{v} \wedge \pi^{*} \eta_{j} \text { is real and }>0 .
\end{gathered}
$$

Let $u$ be a $C R$ function on a connected open neighborhood $V$ of $p_{0}$ in $U^{\prime}$ for the structure defined by (5.2). Since $\mathcal{J}_{M}$ and $\mathcal{J}_{U^{\prime}}^{\prime}$ agree to infinite order on $\pi\left(U^{\prime}\right)$ outside $E=\left\{w_{v} \in \bigcup_{i} \operatorname{supp} \psi_{i}\right\}$, and this set does not disconnect $\pi(V)$, by the argument of Lemma 5.5 we have (5.1) for all $q$ in the complement in $\pi(V)$ of $E$. Thus we obtain

$$
\begin{aligned}
0 & =\int_{\partial K_{j}}\left(\oint_{\pi^{-1}(q)} u d z_{1}\right) d w_{2} \wedge \cdots \wedge d w_{v} \wedge \eta_{j}=\int_{\partial A_{j}} u d z_{1} \wedge \cdots \wedge d z_{v} \wedge \pi^{*} \eta_{j} \\
& =\int_{A_{j}} d u \wedge d z_{1} \wedge \cdots \wedge d z_{v} \wedge \pi^{*} \eta_{j}, \quad \text { yielding }
\end{aligned}
$$

$$
\int_{A_{i}+j(v+1)} \frac{\partial u}{\partial z_{i}} z_{1}^{-1} \psi_{i}\left(z_{v}\right) d \bar{z}_{v} \wedge d z_{1} \wedge \cdots \wedge d z_{v} \wedge \pi^{*} \eta_{i+j(v+1)}=0,
$$

where, to compute $\frac{\partial u}{\partial z_{i}}$, we consider any $\mathcal{C}^{1}$-extension of $u$ as a function of the complex variables $z_{1}, \ldots, z_{v}$ for which $\bar{\partial} u=0$ at all points of $V$. But

$$
c_{i+j(v+1)}^{-1} \int_{A_{i}+j(v+1)} \frac{\partial u}{\partial z_{i}} z_{1}^{-1} \psi_{i}\left(z_{v}\right) d \bar{z}_{v} \wedge d z_{1} \wedge \cdots \wedge d z_{v} \wedge \pi^{*} \eta_{i+j(v+1)}
$$

converges to $\frac{\partial u\left(p_{0}\right)}{\partial z_{i}}$ when $j \rightarrow+\infty$. Therefore $\frac{\partial u\left(p_{0}\right)}{\partial z_{i}}=0$ for $i=1, \ldots, v$. Together with (2.1), this shows that $d u\left(p_{0}\right)=0$.

We have proved:

THEOREM 5.7. Let $M, N$, be CR manifolds of types $(n, k)$ and $(n, k-1)$, respectively, with $k \geq 1$. Assume that $N$ is minimal and that there is a $C R$ map $\pi: M \rightarrow N$ having a Lorentzian $C R$-non characteristic singularity at $p_{0} \in M$. Then we can find a new $C R$ structure of type $(n, k)$ on an open 
neighborhood $U$ of $p_{0}$ in $M$, which agrees with the original one to infinite order at $p_{0}$, such that, if $\mathscr{Z}$ is the distribution of $(0,1)$-vector fields for this new structure, all solutions $u \in \mathcal{C}^{1}$ on a neighborhood of $p_{0}$ to the homogeneous system (2.1) satisfy $d u\left(p_{0}\right)=0$.

Proof. The discussion above proves the theorem in the case where both $M$ and $N$ are locally $C R$-embeddable at $p_{0}$ and at $q_{0}=\pi\left(p_{0}\right)$, respectively. In general, we can reduce to this case by taking formal power series solutions $\{z\}_{1}, \ldots,\{z\}_{v}$ at $p_{0},\{w\}_{2}, \ldots,\{w\}_{v}$ at $q_{0}$, to the homogeneous tangential Cauchy-Riemann systems on $M$ and $N$, respectively, with $\left\{z_{j}\right\}=\pi^{*}\{w\}_{j}$ for $j=2, \ldots, v$. Then we take smooth functions $w_{2}, \ldots, w_{v}$ on $N$ having Taylor series $\{w\}_{2}, \ldots,\{w\}_{v}$ at $q_{0}$, define $z_{j}=\pi^{*} w_{j}$ for $j=2, \ldots, v$, and choose a smooth function $z_{1}$ on $M$ with Taylor series $\left\{z_{1}\right\}$ at $p_{0}$. By restricting to a suitable neighborhood $U$ of $p_{0}$ in $M$ and $W$ of $q_{0}$ in $N$, we obtain $C R$-charts $\left(U ; z_{1}, \ldots, z_{v}\right)$ and $\left(W ; w_{2}, \ldots, w_{v}\right)$ for new $C R$ structures which agree to infinite order with the original ones at $p_{0}$ in $M$ and at $q_{0}$ in $N$. The same map $\pi$ has a Lorentizian $C R$-noncharacteristic singularity at $p_{0}$ also for the new locally $C R$-embeddable $C R$ structures, so that the previous discussion applies.

\section{4 - Example}

Let $n \geq 1, k \geq 1, v=n+k$, and $N$ any $C R$ manifold of type $(n, k-1)$, contained in an open neighborhood $G$ of 0 in $\mathbb{C}^{v-1}$, and minimal. Let $w_{1}, \ldots, w_{v-1}$ be the canonical holomorphic coordinates of $\mathrm{C}^{v-1}$ and assume that $d w_{1}$ and $d \bar{w}_{1}$ are linearly independent on $N$.

Let $\phi: \mathbb{C}^{v} \ni\left(z_{1}, \ldots, z_{v}\right) \rightarrow\left(z_{1}, \ldots, z_{v-1}\right) \in \mathbb{C}^{v-1}$ be the projection onto the first $v-1$ coordinates. If

$$
M=\left\{z \in \mathbb{C}^{v} \mid \phi(z) \in N, \operatorname{Im} z_{1}+\sum_{i=1}^{v-1} z_{i} \bar{z}_{i}=z_{v} \bar{z}_{v}\right\}
$$

then $M$ is a minimal $C R$ submanifold of type $(n, k)$ of $\pi^{-1}(G) \subset \mathbb{C}^{v}$, and the restriction of $\phi$ describes a $C R$ map $\pi: M \rightarrow N$ which has at 0 a Lorentzian $C R$-noncharacteristic singularity.

In particular, there are $C R$ structures on a minimal Lorentzian $C R$ manifold $M$ of arbitrary $C R$ codimension such that a point $p_{0} \in M$ is critical for all $C R$ functions defined on a neighborhood of $p_{0}$. 


\section{Tangential Cauchy-Riemann complexes and a global example}

Throughout this section, $M$ is a smooth $C R$ manifold, of positive $C R$ dimension $n$, and arbitrary $C R$ codimension $k \geq 0$. We set $v=n+k$, and denote by $\mathscr{Z}$ the distribution of smooth complex vector fields of type $(0,1)$ on $M$.

We recall that $\mathcal{O}_{M}(U)$ is the space of $C R$ functions of class $\mathcal{C}^{1}$ on $U^{\text {open }} \subset M$. We set $\mathcal{O}_{M}^{\infty}(U)=\mathcal{O}_{M}(U) \cap \mathcal{C}^{\infty}(U)$, and $\mathcal{O}_{M}^{\infty}(\bar{U})=\mathcal{O}_{M}(U) \cap \mathcal{C}^{\infty}(\bar{U})$ for the restrictions to $\bar{U}$ of smooth functions on $M$, which are $C R$ in $U$. Likewise, when $\Omega$ is an open subset of a complex manifold X, we write $\mathcal{O}(\bar{\Omega})$ for $\mathcal{C}^{\infty}(\bar{\Omega}) \cap \mathcal{O}(\Omega)$.

\section{1 - Definition of the $\bar{\partial}_{M}$-complexes}

Let $\mathcal{J}_{M}$ be the ideal sheaf, corresponding to the characteristic distribution $\mathscr{Z}$ of $(0,1)$-vector fields on $M$. Formal integrability of $\mathscr{Z}$ is equivalent (see Lemma 3.2) to

$$
d \mathcal{J}_{M} \subset \mathcal{J}_{M} .
$$

This implies that also $d\left(\mathcal{J}_{M}\right)^{a} \subset\left(\mathcal{J}_{M}\right)^{a}$, where, for each positive integer $a$, $\left(\mathcal{J}_{M}\right)^{a}$ is the $a$-th exterior power of the ideal $\mathcal{J}_{M}$, and we set $\left(\mathcal{J}_{M}\right)^{0}=\Omega_{M}^{*}$. We can define cochain complexes on $M$ by considering the quotients $\mathscr{2}_{M}^{a, *}=\left(\mathcal{J}_{M}\right)^{a} /\left(\mathcal{J}_{M}\right)^{a+1}$ and the map $\bar{\partial}_{M}: \mathscr{Q}_{M}^{a, *} \rightarrow \mathscr{Q}_{M}^{a, *}$ induced on the quotients by the exterior differential. We have

$$
\mathscr{2}_{M}^{a, *}=\bigoplus_{q \geq 0} \mathscr{2}_{M}^{a, q}, \quad \text { with } \mathscr{Q}_{M}^{a, q}=\left(\left(\mathcal{J}_{M}\right)^{a} \cap \Omega_{M}^{a+q}\right) /\left(\left(\mathcal{J}_{M}\right)^{a+1} \cap \Omega_{M}^{a+q}\right),
$$

and $\bar{\partial}_{M}\left(\mathscr{Q}_{M}^{a, q}\right) \subset \mathscr{2}_{M}^{a, q+1}$. This indeed was the intrinsic definition for the tangential Cauchy-Riemann complexes on $C R$ manifolds given in [33].

Let $\mathcal{Z}^{a, q}(U)=\left\{f \in \mathscr{Q}^{a, q}(U) \mid \bar{\partial}_{M} f=0\right\}$ and $\mathcal{B}^{a, q}(U)=\bar{\partial}_{M}\left(\mathscr{Q}_{M}^{a, q-1}(U)\right)$. The quotient $\mathrm{H}^{a, q}(U)=\mathcal{Z}^{a, q}(U) / \mathcal{B}^{a, q}(U)$ is the cohomology group of the smooth cohomology of $\bar{\partial}_{M}$ on $U$ in bidegree $(a, q)$. We set

$$
\mathrm{H}^{a, q}\left(p_{0}\right)=\lim _{p_{0} \in U^{\text {open }}} \mathrm{H}^{a, q}(U)
$$

for the group of germs of bidegree $(a, q)$-cohomology classes at $p_{0}$.

Let us give a more explicit description of the equations involved in the $\bar{\partial}_{M}$-complexes.

An element of $\mathcal{Z}^{a, q}(U)$ has a representative $f \in \Omega_{M}^{p+q}(U)$. The conditions that $f \in\left(\mathcal{J}_{M}\right)^{a}$ and that its class $[f] \in \mathcal{Z}^{a, q}(U)$ satisfies the integrability 
condition $\bar{\partial}_{M}[f]=0$ are expressed by

$$
\left\{\begin{array}{l}
f \wedge \eta_{1} \wedge \cdots \wedge \eta_{v-a+1}=0, \forall \eta_{1}, \ldots, \eta_{v-a+1} \in \mathcal{J}_{M}^{1}(U) \\
d f \wedge \eta_{1} \wedge \cdots \wedge \eta_{v-a+1}=0, \forall \eta_{1}, \ldots, \eta_{v-a+1} \in \mathcal{J}_{M}^{1}(U)
\end{array}\right.
$$

and the equation $\bar{\partial}_{M} \alpha=[f]$ for $\alpha \in \mathscr{2}^{a, q-1}(U)$ is equivalent to finding $u \in \Omega_{M}^{a+q-1}(U)$ such that

$$
\left\{\begin{array}{l}
u \wedge \eta_{1} \wedge \cdots \wedge \eta_{v-a+1}=0, \forall \eta_{1}, \ldots, \eta_{v-a+1} \in \mathcal{J}_{M}^{1}(U), \\
(d u-f) \wedge \eta_{1} \wedge \cdots \wedge \eta_{v-a+1}=0, \forall \eta_{1}, \ldots, \eta_{v-a+1} \in \mathcal{J}_{M}^{1}(U) .
\end{array}\right.
$$

Both equation (6.2) and (6.3) are meaningful when $f, u$ are currents, and therefore we can consider the $\bar{\partial}_{M}$-complexes on currents, or require different degrees of regularity on the data and the solution.

\section{2 - Absence of Poincaré lemma}

In general, the $\bar{\partial}_{M}$-complexes are not acyclic (see e.g. [3, 4, 18, 33]). In fact, the perturbations we used in the previous section to deduce non complete-integrability results utilize elements of $\mathrm{H}^{0,1}\left(p_{0}\right)$. The arguments of $\S 4$ provide simpler proofs of the absence of the Poincaré lemma in some special cases. We have e.g. (see § 4)

Proposition 6.1. Let $M$ be a CR manifold of type $(n, 1)$, which is locally CR-embeddable and Lorentzian at $p_{0}$, and let $\left(U ; z_{1}, \ldots, z_{v}\right)$, with $v=n+1$, be a CR chart centered at $p_{0}$ for which (4.1) holds and $2 z_{1} \bar{z}_{1} \geq \operatorname{Im} z_{v}$ on $U$.

Let $\psi$ be a smooth function of one complex variable $\tau$, with compact support contained in $\{\operatorname{Im} \tau \geq 0\}$. Then $\omega=z_{1}^{-1} \psi\left(z_{v}\right) d \bar{z}_{v}$, continued by 0 where $z_{1}=0$, defines a smooth $\bar{\partial}_{M}$-closed 1-form. A necessary and sufficient condition for $\omega$ to be cohomologous to 0 is that

$$
\iint \tau^{h} \psi(\tau) d \tau \wedge d \bar{\tau}=0, \forall h \in Z, h \geq 0 .
$$

Proof. Assume indeed that there is $u \in \mathcal{C}^{1}(U)$ such that $\bar{\partial}_{M} u=[\omega]$. We keep the notation of $\S 4$ for $\pi, \omega, B$, and use integration on the fiber to define

$$
g(w)=\frac{-1}{2 \pi i} \oint_{M_{w}} u d z_{1} \text {, i.e. } \int_{\omega} g \phi=\int_{U} u \wedge d z_{1} \wedge \pi^{*} \phi, \quad \forall \phi \in \Omega^{2 n} .
$$


Clearly $g=0$ on $\partial \omega \cap B$ and furthermore we obtain

$$
\begin{aligned}
& \int_{\omega} d g \wedge \eta=\frac{-1}{2 \pi i} \int_{U} d u \wedge d z_{1} \wedge \pi^{*} \eta \\
& =\frac{-1}{2 \pi i} \int z_{1}^{-1} \psi\left(z_{v}\right) d \bar{z}_{v} \wedge d z_{1} \wedge \pi^{*} \eta=\int_{\omega} \psi\left(w_{n}\right) d \bar{w}_{n} \wedge \eta \\
& \forall \eta \in \Omega_{0}^{n-1, n}(\omega) .
\end{aligned}
$$

Thus $g$ satisfies

$$
\begin{cases}\bar{\partial} g=\psi\left(w_{n}\right) d \bar{w}_{n}, & \text { on } \partial \omega, \\ g=0, & \text { on } \partial \omega \cap B .\end{cases}
$$

These equations imply that $\tau \rightarrow g(0, \tau)$ has compact support and hence that the equation $\partial v / \partial \bar{\tau}=\psi(\tau)$ has a solution with compact support. This is equivalent to the momentum conditions (6.4) in the statement.

Wy can repeat the same argument to show that the $\bar{\partial}_{M}$ complexes are not acyclic in dimension $q$ when $M$ is strictly $q$-pseudoconvex at $p_{0}$. Still restraining to type $(n, 1)$ this condition means that, for a suitable $C R$ chart $\left(U ; z_{1}, \ldots, z_{v}\right)$ centered at $p_{0}$ we have

$$
\operatorname{Im} z_{v}+\sum_{i=q+1}^{v} z_{i} \bar{z}_{i}=\sum_{i=1}^{q} z_{i} \bar{z}_{i}+O\left(|z|^{3}\right) \quad \text { on } U .
$$

Here $v=n+1$. By taking $U$ small we can assume that $\operatorname{Im} z_{v} \leq 2 \sum_{i=1}^{q} z_{i} \bar{z}_{i}$ on $U$. Set $\ell=v-q$. By taking $U$ sufficiently small we obtain a proper map

$$
\pi: U \ni p \rightarrow\left(z_{q+1}(p), \ldots, z_{v}(p)\right) \in \omega \subset \mathbb{C}^{\ell},
$$

with $\omega$ the complement of a strictly convex open subset in an open ball $B$ of $\mathrm{C}^{\ell}$, and $M_{w} \sim S^{2 q-1}$ for $w \in \stackrel{\circ}{\omega}$ and $M_{w} \sim$ a point for $w \in \partial \omega \cap B$. Let

$K_{q-1}\left(z_{1}, \ldots, z_{q}\right)=\frac{\sum_{i=1}^{q} \bar{z}_{i} \omega_{i}\left(z_{1}, \ldots, z_{q}\right)}{\left(\sum_{i=1}^{q} z_{i} \bar{z}_{i}\right)^{q}}$ with $\left.\omega_{i}\left(z_{1}, \ldots, z_{q}\right)=\frac{\partial}{\partial \bar{z}_{i}}\right\rfloor d \bar{z}_{1} \wedge \cdots d \bar{z}_{q}$.

Proposition 6.2. Let $M$ be a CR manifold of type $(n, 1)$, which is locally CR-embeddable and strictly q-pseudoconvex at $p_{0}$. Take a CRchart $\left(U, z_{1}, \ldots, z_{v}\right)$ with (6.5), $\operatorname{Im} z_{v} \leq 2 z_{1} \bar{z}_{1}$ on $U$, and let $\pi: M \rightarrow \omega$ be given by (6.6). 
If $\psi$ is a smooth function with compact support of one complex variable $\tau$, with supp $\psi \subset\{\operatorname{Im} \tau \geq 0\}$, then $\omega=\psi\left(z_{v}\right) d \bar{z}_{v} \wedge K_{q-1}\left(z_{1}, \ldots, z_{q}\right)$, continued by 0 where $z_{1}=0$, defines a smooth $\bar{\partial}_{M}$-closed $q$-form on $U$, and (6.4) is a necessary and sufficient condition for $\omega$ to be cohomologous to 0 .

Proof. The proof is analogous to that of Proposition 6.1. Here we need to utilize, instead of Cauchy's formula, the identity

$$
\begin{gathered}
\int_{\pi^{-1}(w)} K_{q-1}\left(z_{1}, \ldots, z_{q}\right) \wedge d z_{1} \wedge \cdots \wedge d z_{q}=\frac{(-1)^{q(q-1) / 2}(q-1) !}{(2 \pi i)^{q}}, \\
\forall w \in \stackrel{\circ}{\circ} \cap \operatorname{supp} \psi\left(w_{\ell}\right) .
\end{gathered}
$$

We have indeed $d\left(K_{q-1}\left(z_{1}, \ldots, z_{q}\right) \wedge d z_{1} \wedge \cdots \wedge d z_{q}\right)=0$ and then the value of the left hand side of (6.7) is a homology invariant. For all $w \in \stackrel{\omega}{\circ}$, the fiber $\pi^{-1}(w)$ is equivalent to the sphere $S^{2 q-1}$. Then the value in (6.7) can be computed by integrating on $S^{2 q-1}$ (see [25]).

\section{3 - Strictly pseudoconvex subdomains of CR manifolds}

Let $\Omega$ be an open set in $M$. Saying that $\partial \Omega$ is smooth at a point $p_{0} \in \partial \Omega$ means that there is an open neighborhood $U$ of $p_{0}$ in $M$ and a smooth real valued function $\phi \in \mathcal{C}^{\infty}(U, \mathbb{R})$ such that

$$
\Omega \cap U=\{p \in U \mid \phi(p) 0\}, \quad d \phi\left(p_{0}\right) \neq 0 .
$$

DeFinition 6.3. We say that $\Omega$ is strictly pseudoconvex at $p_{0}$ if $\mathfrak{L}_{d \phi\left(p_{0}\right)}>0$ on $\boldsymbol{Z}_{p_{0}} M \cap \operatorname{ker} d \phi\left(p_{0}\right)$.

Note that $\boldsymbol{Z}_{p_{0}} M \cap \operatorname{ker} d \phi\left(p_{0}\right)=\boldsymbol{Z}_{p_{0}} M$ when $d \phi\left(p_{0}\right) \in H_{p_{0}}^{0} M$.

We need to introduce a weaker notion of $C R$ function on $M$.

Definition 6.4. If $U^{\text {open }} \subset M$, we say that $u \in \mathcal{C}^{0}(U)$ is $C R$ if

$$
\int_{U} u d \eta=0, \quad \forall \eta \in\left(\mathcal{J}_{M}\right)^{v}(U) \cap \Omega_{M, 0}^{2 n+k-1}(U),
$$

where we indicate by $\Omega_{M, 0}^{*}(U)$ smooth exterior forms having compact support in $U$. 
If $M$ is a $C R$ submanifold of a complex manifold $\mathbb{X}$ and is minimal at $p_{0}$, then all germs of continuous $C R$ functions extend holomorphically to wedges in $\mathbb{X}$ whose edges contain neighborhoods of $p_{0}$ (see $[43,44]$ ). We set $\mathcal{O}_{M}^{\text {cont }}$ for the sheaf of germs of continuous $C R$ functions on $M$. For every $U^{\text {open }} \subset M$, the space $\mathcal{O}_{M}^{\text {cont }}(U)$ is Fréchet for the topology of uniform convergence on the compact subsets of $U$.

DeFinition 6.5. Let $\Omega$ be an open subset of $M, f \in \mathcal{O}_{M}(\Omega)$, and $p_{0} \in \partial \Omega$. We say that $f$ weakly $C R$-extends beyond $p_{0}$ if there exists a connected open neighborhood $U$ of $p_{0}$ in $M$ and $g \in \mathcal{O}_{M}^{\text {cont }}(U)$ such that $\{p \in \Omega \cap U \mid g(p)=f(p)\}$ has a non empty interior.

Proposition 6.6. Let $M$ be a CR submanifold of CR-dimension $n \geq 1$ and arbitrary $C R$ codimension $k \geq 0$ of a Stein manifold $\mathbb{X}$, and $\Omega$ a smooth strictly pseudoconvex domain in X, with $\partial \Omega \cap M \neq \emptyset$. Let $M_{0}$ be the set of points of $M \cap \partial \Omega$ at which $M$ is minimal. Consider on $\mathcal{O}(\bar{\Omega})$ the natural Fréchet topology of uniform convergence with all derivatives on the compact subsets of $\bar{\Omega}$.

Then the set $\mathbb{E}$ of the elements $f \in \mathcal{O}(\bar{\Omega})$ such that $\left.f\right|_{M \cap \bar{\Omega}}$ weakly $C R$ extends beyond some point $p \in M_{0}$ is of the first Baire category in $\mathcal{O}(\bar{\Omega})$.

Proof. Fix a point $p_{0} \in M_{0}$ and a countable fundamental system of open neighborhoods $\left\{V_{v}\right\}_{v \in \mathbb{N}}$ of $p_{0}$ in $\mathbb{X}$. We can assume that for every $v \in \mathbb{N}$ the intersection $M \cap V_{v}$ is connected and contained in the set of minimal points of $M$. In particular, two $C R$ functions on $M \cap V_{v}$ which agree on some non empty open subset of $M \cap V_{v}$, are equal on all $M \cap V_{v}$. For each $v$ the set

$$
\mathrm{F}_{v}=\left\{(f, g) \in \mathcal{O}(\bar{\Omega}) \times \mathcal{O}_{M}^{\text {cont }}\left(M \cap V_{v}\right) \mid g=f \text { on } M \cap \Omega \cap V_{v}\right\}
$$

is a Fréchet space, being a closed subspace of $\mathcal{O}(\bar{\Omega}) \times \mathcal{O}_{M}^{\text {cont }}\left(M \cap V_{v}\right)$.

Let $\pi_{v}: \mathbb{F}_{v} \rightarrow \mathcal{O}(\bar{\Omega})$ be the projection into the first component.

Assume by contradiction that the set of $f \in \mathcal{O}(\bar{\Omega})$ such that $\left.f\right|_{M \cap \Omega}$ can be weakly $C R$-continued beyond $p_{0}$ is of the second Baire category. Then $\bigcup_{v \in \mathbb{N}} \pi_{v}\left(\mathbb{F}_{v}\right)$ is of the second Baire category, and hence at least one $\pi_{v_{0}}\left(\mathbb{F}_{v_{0}}\right)$ is of the second Baire category in $\mathcal{O}(\bar{\Omega})$. Hence $\pi_{v_{0}}\left(\mathbb{F}_{v_{0}}\right)=\mathcal{O}(\bar{\Omega})$ and, by Banach-Shauder's theorem, the map $\pi_{v_{0}}: \boldsymbol{F}_{v_{0}} \rightarrow \mathcal{O}(\bar{\Omega})$ is open (see e.g. $[40, \S 2.1])$. By the assumption of minimality and the fact that Lemma 5.1 also applies to continuous $C R$ functions, for each $f \in \mathcal{O}(\bar{\Omega})$ there is at most one $g \in \mathcal{O}_{M}^{\text {cont }}\left(M \cap V_{v}\right)$ such that $(f, g) \in \mathbb{F}_{v}$. 
Thus, we conclude that there is a relatively compact open neighborhood $V$ of $p_{0}$ in $\mathbb{X}$ such that

$$
\begin{gathered}
\forall f \in \mathcal{O}(\bar{\Omega}) \quad \exists ! g \in \mathcal{O}_{M}(M \cap V) \quad \text { with } f=g \text { on } M \cap \Omega \cap V \text { and } \\
|g(p)| \leq\|f\|_{\ell, K}, \quad \forall p \in M \cap V,
\end{gathered}
$$

where $K$ is a compact subset of $\bar{\Omega}$ and $\|f\|_{\ell, K}$ a seminorm involving the derivatives of $f$ up to order $\ell$ on $K$. Let $K_{v}$ be a sequence of compact subsets of $\mathbb{X}$ such that $K_{v+1} \Subset \operatorname{int}\left(K_{v}\right)$, for all $v \in \mathbb{N}$, and $\bigcap_{v} K_{v}=K$. By Cauchy's inequalities, there are constants $C_{v}>0$ such that

$$
\|f\|_{\ell, K} \leq C_{v} \sup _{K_{v}}|f|, \quad \forall f \in \mathcal{O}(\mathbb{X}) .
$$

Using (6.10) we obtain

$$
|f(p)| \leq C_{v} \sup _{K_{v}}|f|, \quad \forall f \in \mathcal{O}(\mathbb{X}), \quad \forall p \in M \cap V .
$$

By applying this inequality to the positive integral powers of the entire functions on $\mathbb{X}$, we obtain that

$$
\begin{gathered}
|f(p)| \leq C_{v}^{1 / h} \sup _{K_{v}}|f|, \quad \forall f \in \mathcal{O}(\mathbb{X}), \quad \forall p \in M \cap V, \quad \forall 0<h \in \mathbb{N} \\
\Longrightarrow|f(p)| \leq \sup _{K_{v}}|f|, \quad \forall f \in \mathcal{O}(\mathbb{X}), \quad \forall p \in M \cap V .
\end{gathered}
$$

Since $\bigcap_{v} K_{v}=K$, we obtain

$$
|f(p)| \leq \sup _{K}|f|, \quad \forall f \in \mathcal{O}(\mathbb{X}) .
$$

But this gives a contradiction, because the fact that $\Omega$ is strictly pseudoconvex implies that $\bar{\Omega}$ is holomorphically convex in $\mathbb{X}$.

We showed that, for every point $p \in M_{0}$, the set $\mathbb{E}_{p}$ of $f \in \mathcal{O}(\bar{\Omega})$ for which $\left.f\right|_{M \cap \Omega}$ weakly $C R$-extends beyond $p$ is of the first Baire category in $\mathcal{O}(\bar{\Omega})$. The set $M_{0}$ is separable. Then we take a dense sequence $\left\{p_{v}\right\}$ in $M_{0}$ and we observe that $\mathbb{E}=\bigcup_{v \in \mathbb{N}} \mathbb{E}_{p_{v}}$ is of the first Baire category, being a countable union of sets of the first Baire category.

\section{4 - The Cauchy problem for $\bar{\partial}_{M}$}

Let $F^{\text {closed }}, U^{\text {open }} \subset M$. Set, for all $a=0, \ldots, v, q=0, \ldots, n$,

$$
\mathscr{Q}^{a, q}(U ; F)=\left\{f \in \mathscr{Q}^{a, q}(U) \mid f=0^{\infty} \text { on } F \cap U\right\} .
$$


Clearly $\left(\mathscr{Q}^{a, *}(U ; F), \bar{\partial}_{M}\right)$ is a subcomplex of $\left(\mathscr{Q}^{a, *}(U), \bar{\partial}_{M}\right)$ and we can consider the cohomology groups

$$
\mathrm{H}^{a, q}(U, F)=\operatorname{ker}\left(\bar{\partial}_{M}: \mathscr{Q}^{a, q}(U ; F) \rightarrow \mathscr{2}^{a, q+1}(U ; F)\right) / \bar{\partial}_{M} \mathscr{Q}^{a, q-1}(U ; F)
$$

and also their germs

$$
\mathrm{H}^{a, q}\left(p_{0}, F\right)=\lim _{p_{0} \in U^{\text {open }}} \mathrm{H}^{a, q}(U, F) .
$$

We obtain from Proposition 6.6

Proposition 6.7. Let $M$ be a $C R$ submanifold of positive $C R$ dimension $n$ of a Stein manifold $\mathbb{X}$. Let $M^{\prime}$ be the set of points of $M$ where $M$ is minimal. Let $\Omega$ be a strictly pseudoconvex open subset of $\mathbb{X}$, with $M_{0}=M^{\prime} \cap \partial \Omega \neq \emptyset$. Then the set of $\alpha \in \mathrm{H}^{0,1}(M, \bar{\Omega} \cap M)$ that restrict to the zero class of $\mathrm{H}^{0,1}(p, \bar{\Omega} \cap M)$ for some $p \in M_{0}$ is infinite dimensional.

More precisely, if we consider the Fréchet space

$$
\mathscr{F}=\left\{f \in \Omega^{1}(M)|f|_{\bar{\Omega} \cap M}=0^{\infty}, \quad d f \in \mathcal{J}_{M}\right\},
$$

then the set of $f \in \mathscr{F}$ for which we can find $p \in M_{0}$ and $u_{(p)} \in \mathcal{C}_{(p)}^{0}$ with $u_{(p)}$ vanishing on $\bar{\Omega} \cap M$, and $\bar{\partial}_{M} u_{(p)}=f_{(p)}$, span a linear subspace of infinite dimensional codimension in $\mathscr{F}$. Here we wrote $f_{(p)}$ for the germ of $f$ at $p$, and the equality $\bar{\partial}_{M} u_{(p)}=f_{(p)}$ has to be interpreted in the weak sense: it means that there is an open neighborhood $U_{p}$ of $p$ and a continuous function $u \in \mathcal{C}\left(U_{p}\right)$, vanishing on $\bar{\Omega} \cap U_{p}$, such that

$$
\int_{M} u d \eta=-\int_{M} f \wedge \eta, \quad \forall \eta \in\left(\mathcal{J}_{M}\right)^{v}\left(U_{p}\right) \cap \Omega_{0}^{2 n+k-1}\left(U_{p}\right) .
$$

Proof. Let $g \in \mathcal{O}(\bar{\Omega})$. By Whitney's extension theorem, there is a smooth complex valued function $\tilde{g}$ on $\mathbb{X}$ with $\tilde{g}=g$ on $\bar{\Omega}$. Then $f=\left.\bar{\partial} \tilde{g}\right|_{M}$ is an element of $\mathscr{F}$. Let $w=\left.\tilde{g}\right|_{M}$. Then $w$ is a smooth function on $M$ and its restriction to $M \cap \Omega$ is $C R$. If $p \in M_{0}$ and $u_{(p)} \in \mathcal{C}_{(p)}^{0}$ solves $\bar{\partial}_{M} u_{(p)}=f_{(p)}$, then $w_{(p)}+u_{(p)}$ yields a weak $C R$ extension of $\left.w\right|_{M \cap \Omega}$ beyond $p$. Therefore the thesis follows by Proposition 6.6.

Let $N$ be a smooth submanifold of $M$. Its conormal bundle in $M$ at $p \in N$ is

$$
T_{N, p}^{*} M=\left\{\xi \in T_{p}^{*} M \mid \xi(v)=0, \forall v \in T_{p} N\right\} .
$$

Definition 6.8. We say that $N$ is characteristic at $p \in N$ if $T_{N, p}^{*} M \cap H_{p}^{0} M \neq\{0\}$. 
Equivalently, $N$ is non characteristic at $p \in N$ if it contains a germ $\left(N^{\prime}, p\right)$ of $C R$ submanifold of type $(0, v)$ of $M$. We have the following

LEMMA 6.9. Let $M$ be a $C R$ manifold of positive $C R$ dimension $n$, and $N$ a smooth submanifold of $M$. Let $U$ be an open neighborhood of a non characteristic point $p_{0}$ of $N$.

If $\in \mathscr{2}^{0,1}(U, N)$ and $u \in \mathcal{C}^{\infty}(U)$ solves

$$
\begin{cases}\bar{\partial}_{M} u=f & \text { on } U, \\ u=0 & \text { on } N\end{cases}
$$

then $u$ vanishes to infinite order at $p_{0}$.

Proof. The statement follows by the uniqueness in the formal non characteristic Cauchy problem.

\section{5 - The canonical bundle}

The sheaf $\mathscr{2}^{v, 0}=\left(\mathcal{J}_{M}\right)^{v} \cap \Omega_{M}^{v}$ is the sheaf of germs of smooth sections of a line bundle $K M$ on $M$, that is called the canonical bundle of $M$.

Let $U^{\text {open }} \subset M$, and assume that $\theta_{1}, \ldots, \theta_{v} \in \mathcal{J}_{M}(U)$ give a basis of $\boldsymbol{Z}_{p}^{0} M$ at all points $p \in U$. Since $d \theta_{j} \in \mathcal{J}_{M}(U)$, we obtain

$$
d\left(\theta_{1} \wedge \cdots \wedge \theta_{v}\right)=\alpha \wedge \theta_{1} \wedge \cdots \wedge \theta_{v}, \text { with } \alpha \in \Omega^{1}(U) .
$$

The form $\alpha$ is uniquely determined modulo $\mathcal{J}_{M}$, and thus defines a unique element $[\alpha] \in \mathscr{2}^{0,1}(U)$. By differentiating we get

$$
\begin{aligned}
0 & =d^{2}\left(\theta_{1} \wedge \cdots \wedge \theta_{v}\right)=(d \alpha) \wedge \theta_{1} \wedge \cdots \wedge \theta_{v}-\alpha \wedge d\left(\theta_{1} \wedge \cdots \wedge \theta_{v}\right) \\
& =(d \alpha) \wedge \theta_{1} \wedge \cdots \wedge \theta_{v}-\alpha \wedge \alpha \wedge \theta_{1} \wedge \cdots \wedge \theta_{v} \\
& =(d \alpha) \wedge \theta_{1} \wedge \cdots \wedge \theta_{v} \Longleftrightarrow \bar{\partial}_{M}[\alpha]=0 .
\end{aligned}
$$

If $\omega$ is another non zero section of $K M$, defined in a neighborhood of a point $p_{0} \in U$, we have $\omega=e^{u} \theta_{1} \wedge \cdots \wedge \theta_{v}$ on a neighborhood $U_{p_{0}}$ of $p_{0}$ in $U$ and hence

$$
d \omega=e^{u}(d u+\alpha) \wedge \theta_{1} \wedge \cdots \wedge \theta_{v} \text { on } U_{p_{0}} .
$$

Thus we obtain

Lemma 6.10. There is a section $\psi \in \Gamma\left(M, H^{0,1}\right)$ of the sheaf of germs of cohomology classes of bidegree $(0,1)$ such that 


$$
\left\{\begin{array}{c}
\forall p \in M, \quad \forall \omega \in \Gamma_{(p)}(K M), \text { with } \omega(p) \neq 0, \quad \exists \alpha \in \Omega_{(p)}^{1} \text { such that } \\
d \omega(p)=\alpha(p) \wedge \omega(p) . \bar{\partial}_{M}[\alpha]=0, \llbracket \alpha \rrbracket \in \psi(p)
\end{array}\right.
$$

Here $\llbracket \alpha \rrbracket$ is the element of $\mathrm{H}^{0,1}(p)$ defined by $[\alpha] \in \mathcal{Z}_{(p)}^{0,1}$.

LEMma 6.11. If $M$ is locally CR-embeddable at $p$, then $\psi(p)=0$.

If $\mathscr{Z}$ is completely integrable at $p$, then, for $\alpha \in \Omega_{(p)}^{1}$ satisfying (6.12) there is $u \in \mathcal{C}_{(p)}^{0}$ such that $\bar{\partial}_{M} u=[\alpha]$.

\section{$6.6-C R$-foldings}

Let us recall the notion of a fold singularity for a smooth map (see e.g. [13]). Let $M, N$ be real smooth manifolds of the same real dimension. A map $\pi: M \rightarrow N$ is a fold-map if there is a smooth submanifold $V$ of $M$ such that:

- the restriction of $\pi$ to $M \backslash V$ is a two-sheeted covering;

- the restriction of $\pi$ to $V$ is a smooth immersion;

- there is an involution $\sigma: U \rightarrow U$ of a tubular neighborhood $U$ of $V$ in $M$ such that $\pi \circ \sigma=\pi$ on $U$.

The corresponding notion in $C R$ geometry will be a folding about a $C R$-divisor. Let us introduce the notions that we will utilize in the sequel.

Definition 6.12 (Smooth $C R$-divisors). $A$ smooth $C R$-divisor of a $C R$ manifold $M$ is a smooth submanifold $D$ of $M$, having real codimenison 2 , such that for each $p_{0} \in D$ there is an open neighborhood $U_{p_{0}}$ of $p_{0}$ in $M$ and a function $f \in \mathcal{O}_{M}^{\infty}\left(U_{p_{0}}\right)$ such that $D \cap U_{p_{0}}=\left\{p \in U_{p_{0}} \mid f(p)=0\right\}$ and $d f\left(p_{0}\right) \wedge d \bar{f}\left(p_{0}\right) \neq 0$.

Definition 6.13 (CR-folding). Let $M, N$ be $C R$ manifolds having the same CR dimension. A CR-folding is a proper smooth $C R$ map $\pi: M \rightarrow N$ such that there exists a smooth $C R$-divisor $D$ on $M$ with the properties:

- the restriction of $\pi$ to $M \backslash D$ is a CR-submersion and a smooth circle bundle;

- the restriction of $\pi$ to $D$ is a smooth CR immersion;

- there is a tubular neighborhood $U$ of $D$ in $M$ and an $\boldsymbol{S}^{1}$-action on the fibers of $\left.\pi\right|_{U}: U \rightarrow \pi(U)$, for which $D$ is the set of fixed points. 
ExAMPle 6.14. The map $S^{3}=\{(z, w) \mid z \bar{z}+w \bar{w}=1\} \ni(z, w) \stackrel{\pi}{\longrightarrow} z \in \mathbb{C}$ is a $C R$-folding. The set $D=\{(z, 0) \mid z \bar{z}=1\}=\left\{(z, w) \in S^{3} \mid w=0\right\}$ is a smooth $C R$-divisor in $S^{3}$, and $\pi^{-1}\left(z_{0}\right)=\left\{\left(z_{0}, w\right) \mid w \bar{w}=1-z_{0} \bar{z}_{0}\right\}$. We can define, in the tubular neighborhood $U=S^{3} \cap\{|z|>0\}$, an $\boldsymbol{S}^{1}$-action by $e^{i \theta} \cdot(z, w)=\left(z, e^{i \theta} w\right)$.

ExAmPLE 6.15. Let $Q \subset \mathbb{C P}^{v}$, with $v \geq 3$, be the ruled real projective quadric, which is a $C R$ submanifold of type $(n, 1)$, with $n=v-1$, and Levi signature $(1, n-1)$. For a suitable choice of homogeneous coordinates, $Q$ has equation

$$
z_{0} \bar{z}_{0}+z_{1} \bar{z}_{1}=\sum_{j=2}^{v} z_{j} \bar{z}_{j} .
$$

The point $p_{0} \equiv(1,0, \ldots, 0)$ does not belong to $Q$. Hence, by associating to each $p \in Q$ the complex line $p_{0} p$, we obtain a map $\pi: Q \rightarrow \mathbb{C P}^{n}$, where $\mathrm{CP}^{n}$ is the set of complex lines through $p_{0}$ of $\mathrm{CP}^{n+1}$. After identifying $\mathrm{CP}^{n}$ with the hyperplane $\left\{z_{0}=0\right\}$, the map $\pi$ is described in homogeneous coordinates by

$$
\pi:\left(z_{0}, z_{1}, \ldots, z_{v}\right) \longrightarrow\left(z_{1}, \ldots, z_{v}\right) .
$$

This map is a $C R$-folding of $M$ into $\boldsymbol{C P} \boldsymbol{P}^{n}$, with divisor $Q \cap\left\{z_{0}=0\right\}$, whose image is the complement of an open ball in the projective space:

$$
\pi(Q)=\left\{z_{1} \bar{z}_{1} \leq \sum_{j=2}^{v} z_{j} \bar{z}_{j}\right\} .
$$

To describe the generators of the ideal sheaf $\mathcal{J}_{Q}$ of $Q$, we consider the covering $\{U, V\}$ of $Q$ with $U=\left\{z_{0} \neq 0\right\}, V=\left\{z_{1} \neq 0\right\}$. Then $\mathcal{J}_{Q}$ is defined by the generators

$$
\begin{array}{ll}
z_{0}^{-1} d z_{1}, z_{0}^{-1} d z_{2}, \ldots, z_{0}^{-1} d z_{v} & \text { on } U \cap Q, \\
z_{1}^{-1} d z_{0}, z_{1}^{-1} d z_{2}, \ldots, z_{1}^{-1} d z_{v} & \text { on } V \cap Q .
\end{array}
$$

The canonical bundle is generated by $z_{0}^{-v} d z_{1} \wedge d z_{2} \wedge \cdots d z_{v}$ on $U \cap Q$ and by $z_{1}^{-v} d z_{0} \wedge d z_{2} \wedge \cdots d z_{v}$ on $V \cap Q$.

\section{7 - Construction of a locally non CR-embeddable perturbation}

In this subsection we describe a procedure to define a non locally $C R$ embeddable $C R$ structure on a neighborhood of $p_{0}$ in $M$ that we will use in $\S 6.8$ to produce a global example. 
Let $M, N$ be $C R$ manifolds of type $(n, k),(n, k-1)$ respectively. Assume that there is a $C R$ map $\pi: M \rightarrow N$ having a Lorentzian singularity and a $C R$-folding at $p_{0}$, and that $M, N$ are locally $C R$-embeddable at $p_{0}, q_{0}$, respectively. We are in fact in the situation of Lemma 5.4 and we keep the notation therein.

Let $\alpha$ be a $(0,1)$-form on $\omega$, with $\bar{\partial}_{N} \alpha=0$ and $\alpha=0$ on $\omega_{+}$. Then $z_{1}^{-1} \pi^{*} \alpha$ is well defined and smooth because $\pi^{*} \alpha$ vanishes to infinite order on $D=\left\{z_{1}=0\right\}$, and we may consider on $U$ the $C R$-structure with ideal sheaf $\mathcal{J}_{U}^{\prime}$ generated by

$$
d z_{1}-z_{1}^{-1} \pi^{*} \alpha, d z_{2}, \ldots, d z_{v} .
$$

This new $C R$ structure agrees with the original one to infinite order at all points of $D$. If this new $C R$ structure admits a $C R$-chart centered at $p_{0}$, then there is a smooth function $u$, defined on a neighborhood of $p_{0}$, with $u\left(p_{0}\right)=0$ and

$$
d\left(e^{u}\left(d z_{1}-z_{1}^{-1} \pi^{*} \alpha\right) \wedge d z_{2} \wedge \cdots \wedge d z_{v}\right)=0 .
$$

Then we obtain

$$
d u \wedge\left(d z_{1}-z_{1}^{-1} \pi^{*} \alpha\right) \wedge d z_{2} \wedge \cdots \wedge d z_{v}+z_{1}^{-2} d z_{1} \wedge \pi^{*} \alpha \wedge d z_{2} \wedge \cdots \wedge d z_{v}=0,
$$

from which we get

$$
\left(d\left(z_{1}^{2} u\right)-\pi^{*} \alpha^{*}\right) \wedge \frac{d z_{1}}{z_{1}} \wedge d z_{2} \wedge \cdots \wedge d z_{v}+d\left(u \pi^{*} \omega^{*} \wedge d z_{2} \wedge \cdots \wedge d z_{v}\right)=0 .
$$

Next we integrate on the fiber. For $q \in W \cap \omega_{-}$, where $W$ is a suitable small neighborhood of $q_{0}$ in $\omega$, we obtain

$$
w(q)=\pi_{*}\left(z_{1}^{2} u\right)(q)=\frac{1}{2 \pi i} \oint_{\pi^{-1}(q)} z_{1} u d z_{1},
$$

and therefore

$$
\begin{aligned}
& d w(q) \wedge d z_{2} \wedge \cdots \wedge d z_{v}=\frac{1}{2 \pi i} \oint_{\pi^{-1}(q)} d\left(z_{1}^{2} u\right) \frac{d z_{1}}{z_{1}} \wedge d z_{2} \wedge \cdots \wedge d z_{v} \\
& =\frac{1}{2 \pi i} \oint_{\pi^{-1}(q)} \pi^{*} \alpha^{*} \frac{d z_{1}}{z_{1}} \wedge d z_{2} \wedge \cdots \wedge d z_{v}=\alpha \wedge d z_{2} \wedge \cdots \wedge d z_{v},
\end{aligned}
$$

because

$$
\oint_{\pi^{-1}(q)} d\left(u \pi^{*} \omega^{*} \wedge d z_{2} \wedge \cdots \wedge d z_{v}\right)=0
$$


We observe that $w=0^{2}$ on $W \cap \partial \omega_{-}$and that the equality established above means that $w$ satisfies

$$
\bar{\partial}_{N} w=[\alpha] \text { on } N .
$$

By Lemma 6.9, we actually have $w=0^{\infty}$ on $\partial N$. By Proposition 6.7 there are $\bar{\partial}_{N}$-closed forms $\alpha$, of type $(0,1)$, vanishing to infinite order on $W \cap \partial \omega_{-}$, for which the Cauchy problem

$$
\begin{cases}\bar{\partial}_{N} w=\alpha & \text { on } W \cap \omega_{-}, \\ w=0^{\infty} & \text { on } W \cap \partial \omega_{-}\end{cases}
$$

has no solution when $W$ is any open neighborhood of $q_{0}$, hence yielding non $C R$-embeddable $C R$ structures on $U$ which agree to infinite order with the original one.

\section{8 - A global example}

In this section we construct a non locally $C R$-embeddable $C R$ structure on the Lorentzian quadric of Example 6.15.

In $\mathrm{CP}^{v}$, with homogeneous coordinates $z_{0}, z_{1}, \ldots, z_{v}$, for $v \geq 2$, we consider the quadric $Q=\left\{z_{0} \bar{z}_{0}+z_{1} \bar{z}_{1}=z_{2} \bar{z}_{2}+\cdots+z_{v} \bar{z}_{v}\right\}$. Fix the divisor $D=\left\{z_{0}=0\right\}$ and the global $C R$-folding $Q \rightarrow N$ where $N=$ $\left\{z_{1} \bar{z}_{1} \leq z_{2} \bar{z}_{2}+\cdots+z_{v} \bar{z}_{v}\right\} \subset \mathrm{CP}^{v-1}$ is a strictly pseudoconcave closed domain in $\mathrm{CP}^{\mathrm{v}-1}$. The closure of its complement is an Euclidean ball $B$ of $\mathrm{C}^{v-1}=\mathrm{CP}^{v-1} \backslash\left\{z_{1}=0\right\}$. Fix a smooth function $f$, with compact support in $\mathrm{C}^{v-1}$, which is holomorphic on $B$, but cannot be continued holomorphically beyond any point of $\partial B$, and let $\alpha$ be the restriction of $\bar{\partial} f$ to $N$. We observe that $\zeta=\frac{z_{1}}{z_{0}}$ is meromorphic on $\boldsymbol{C} \boldsymbol{P}^{m}$ and that $\zeta \pi^{*} \alpha$ is well-defined on $Q$. We define a new $C R$ structure on $Q$ by the ideal sheaf having generators

$$
\begin{gathered}
z_{1}^{-1} d z_{0}-\zeta \pi^{*} \alpha^{*}, z_{1}^{-1} d z_{2}, \ldots, z_{1}^{-1} d z_{v} \quad \text { on } M \cap\left\{z_{1} \neq 0\right\}, \\
z_{0}^{-1} d z_{1}, z_{0}^{-1} d z_{2}, \ldots, z_{0}^{-1} d z_{v} \quad \text { on } M \backslash \operatorname{supp} \pi^{*} \alpha .
\end{gathered}
$$

Note that the hyperplane $\left\{z_{1}=0\right\}$ in $\mathrm{CP}^{v-1}$ does not intersect the support of $\alpha$, and hence the ideal sheaf is well defined. By the argument in $\S 6.7$, with this new $C R$ structure $Q$ is not locally $C R$-embeddable at all points of the divisor $D$. Thus we have obtained 
THEOREM 6.16. There are $C R$ structures of type $(m-1,1)$ on the Lorentzian quadric $Q$ that are not locally $C R$-embeddable at all points of a hyperplane section of $Q$.

\section{REFERENCES}

[1] TAKao Akahori, A new approach to the local embedding theorem of CRstructures for $n \geq 4$ (the local solvability for the operator $\bar{\partial}_{b}$ in the abstract sense), Mem. Amer. Math. Soc. 67, no. 366 (1987), pp. xvi+257.

[2] Andrea Altomani - C. Denson Hill - Mauro Nacinovich - Egmont Porten, Complex vector fields and hypoelliptic partial differential operators, Ann. Inst. Fourier (Grenoble), 60, no. 3 (2010), pp. 987-1034.

[3] Aldo Andreotti - Gregory Fredricks - Mauro Nacinovich, On the absence of Poincaré lemma in tangential Cauchy-Riemann complexes, Ann. Scuola Norm. Sup. Pisa Cl. Sci. (4) 8, no. 3 (1981), pp. 365-404.

[4] Aldo Andreotti - C. Denson Hill, E. E. Levi convexity and the Hans Lewy problem. II. Vanishing theorems, Ann. Scuola Norm. Sup. Pisa (3) 26 (1972), pp. 747-806.

[5] M. S. BAOUENDI - L. P. RoTHSCHILD, Embeddability of abstract CR structures and integrability of related systems, Ann. Inst. Fourier (Grenoble), 37, no. 3 (1987), pp. 131-141.

[6] M. S. BaOUendi - Linda Preiss Rothschild, Cauchy-Riemann functions on manifolds of higher codimension in complex space, Invent. Math. 101, no. 1 (1990), pp. 45-56.

[7] M. S. BAOUENDI - F. TRÈVEs, A property of the functions and distributions annihilated by a locally integrable system of complex vector fields, Ann. of Math. (2), 113, no. 2 (1981), pp. 387-421.

[8] Shiferaw Berhanu - Paulo D. Cordaro - Jorge Hounie, An introduction to involutive structures, New Mathematical Monographs, vol. 6, Cambridge University Press, Cambridge, 2008.

[9] L. Boutet DE Monvel, Intégration des équations de Cauchy-Riemann induites formelles, Séminaire Goulaouic-Lions-Schwartz 1974-1975; Équations aux derivées partielles linéaires et non linéaires, Centre Math., École Polytech., Paris, 1975, pp. Exp. No. 9, 14.

[10] J. Brinkschulte - C. Denson Hill - M. Nacinovich, Obstructions to generic embeddings, Ann. Inst. Fourier (Grenoble), 52, no. 6 (2002), pp. 1785-1792.

[11] Judith Brinkschulte - C. Denson Hill - Mauro Nacinovich, The Poincaré lemma and local embeddability, Boll. Unione Mat. Ital. Sez. B Artic. Ric. Mat. (8), 6, no. 2 (2003), pp. 393-398.

[12] David Catlin, Sufficient conditions for the extension of CR structures, J. Geom. Anal. 4, no. 4 (1994), pp. 467-538.

[13] JA. M. ÈLIAŠBERG, Singularities of folding type, Izv. Akad. Nauk SSSR Ser. Mat. 34 (1970), pp. 1110-1126.

[14] C. Denson Hill, What is the notion of a complex manifold with a smooth boundary?, Algebraic analysis, Vol. I, Academic Press (Boston, MA, 1988), pp.185-201. 
[15] C. Denson Hill, Counterexamples to Newlander-Nirenberg up to the boundary, Several complex variables and complex geometry, Part 3 (Santa Cruz, CA, 1989), Proc. Sympos. Pure Math., vol. 52, Amer. Math. Soc., Providence (RI, 1991), pp. 191-197.

[16] C. Denson Hill - Mauro Nacinovich, Embeddable CR manifolds with nonembeddable smooth boundary, Boll. Un. Mat. Ital. A (7), 7, no. 3 (1993), pp. 387-395.

[17] C. Denson Hill - Mauro Nacinovich, Solvable Lie algebras and the embedding of CR manifolds, Boll. Unione Mat. Ital. Sez. B Artic. Ric. Mat. (8), 2, no. 1 (1999), pp. 121-126.

[18] C. Denson Hill - Mauro Nacinovich, On the failure of the Poincaré lemma for $\bar{\partial}_{M}$. II, Math. Ann. 335, no. 1 (2006), pp. 193-219.

[19] LARS HöRMANDER, On existence of solutions of partial differential equations, Partial differential equations and continuum mechanics, Univ. of Wisconsin Press, Madison, Wis. (1961), pp. 233-240.

[20] Howard JacoBOWITz, Simple examples of nonrealizable CR hypersurfaces, Proc. Amer. Math. Soc. 98, no. 3 (1986), pp. 467-468.

[21] Howard JacoBowitz, Homogeneous solvability and CR structures, Notas de Curso [Course Notes], vol. 25, Universidade Federal de Pernambuco Departamento de Matemática, Recife, 1988.

[22] Howard JacoBowitz - FrançoIs TrÈves, Nonrealizable CR structures, Invent. Math. 66, no. 2 (1982), pp. 231-249.

[23] Howard Jacobowitz - FrançoIs TrÈves, Nowhere solvable homogeneous partial differential equations, Bull. Amer. Math. Soc. (N.S.), 8, no. 3 (1983), 467-469.

[24] Howard JacoBowitz - FrançoIs TRÈVEs, Aberrant CR structures, Hokkaido Math. J. 12 (1983), pp. 276-292.

[25] Walter Koppelman, The Cauchy integral for functions of several complex variables, Bull. Amer. Math. Soc. 73 (1967), pp. 373-377.

[26] Masatake KuRAnishi, Strongly pseudoconvex CR structures over small balls. I. An a priori estimate, Ann. of Math. (2), 115, no. 3 (1982), pp. 451-500.

[27] Masatake KURANishi, Strongly pseudoconvex CR structures over small balls. II. A regularity theorem, Ann. of Math. (2), 116, no. 1 (1982), pp. 1-64.

[28] Masatake KuRANishi, Strongly pseudoconvex CR structures over small balls. III. An embedding theorem, Ann. of Math. (2), 116, no. 2 (1982), pp. 249-330.

[29] Hans LEwy, On the local character of the solutions of an atypical linear differential equation in three variables and a related theorem for regular functions of two complex variables, Ann. of Math. (2), 64 (1956), pp. 514-522.

[30] Hans Lewy, An example of a smooth linear partial differential equation without solution, Ann. of Math. (2), 66 (1957), pp. 155-158.

[31] LAN Ma - JOACHIM Michel, Regularity of local embeddings of strictly pseudoconvex CR structures, J. Reine Angew. Math. 447 (1994), pp. 147-164.

[32] Abdelhamid Meziani, Perturbation of a class of $C R$ structures of codimension larger than one, J. Funct. Anal. 116, no. 1 (1993), pp. 225-244.

[33] M. Nacinovich, Poincaré lemma for tangential Cauchy-Riemann complexes, Math. Ann. 268, no. 4 (1984), pp. 449-471.

[34] M. Nacinovich - E. Porten, $\mathcal{C}^{\infty}$-hypoellipticity and extension of cr functions, arXiv:1107.3374 (2011). 
[35] A. Newlander - L. Nirenberg, Complex analytic coordinates in almost complex manifolds, Ann. of Math. (2), 65 (1957), pp. 391-404.

[36] Louis Nirenberg, A certain problem of Hans Lewy, Uspehi Mat. Nauk, 29, no. 2(176) (1974), pp. 241-251, Translated from the English by Ju. V. Egorov, Collection of articles dedicated to the memory of Ivan Georgievič Petrovski (1901-1973), I.

[37] Louis Nirenberg - François TRÈves, On local solvability of linear partial differential equations. I. Necessary conditions, Comm. Pure Appl. Math. 23 (1970), pp. 1-38.

[38] Louis Nirenberg - François Trèves, On local solvability of linear partial differential equations. II. Sufficient conditions, Comm. Pure Appl. Math. 23 (1970), pp. 459-509.

[39] S. I. PINCHUK - S. V. KHASANOv, Asymptotically holomorphic functions and their applications, Mat. Sb. (N.S.), 134(176), no. 4 (1987), pp. 546-555, 576.

[40] H. H. Schaefer - M. P. WolfF, Topological vector spaces, second ed., Graduate Texts in Mathematics, vol. 3, Springer-Verlag, New York, 1999.

[41] F. TRÈVES, Approximation and representation of functions and distributions annihilated by a system of complex vector fields, École Polytechnique Centre de Mathématiques, Palaiseau, 1981.

[42] François TrÈves, Hypo-analytic structures, Princeton Mathematical Series, vol. 40, Princeton University Press, Princeton, NJ, 1992, Local theory.

[43] A. E. Tumanov, Extension of CR-functions into a wedge from a manifold of finite type, Mat. Sb. (N.S.), 136(178), no. 1 (1988), pp. 128-139.

[44] A. E. Tumanov, Extension of CR-functions into a wedge, Mat. Sb. 181, no. 7 (1990), pp. 951-964.

Manoscritto pervenuto in redazione il 7 Novembre 2011. 
